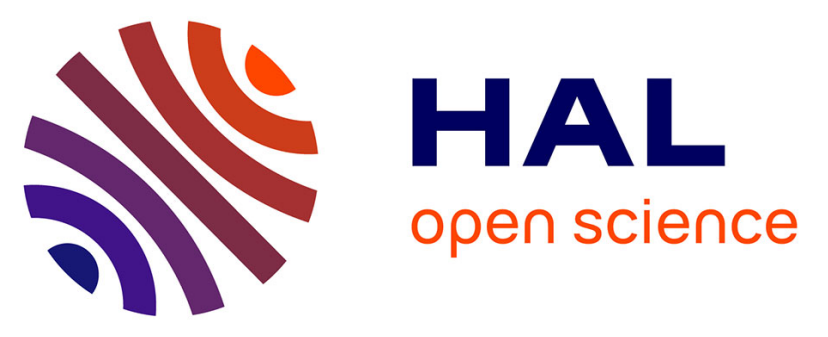

\title{
Les élections régionales du 15 mars 1998 en Languedoc-Roussillon. Une nouvelle singularité politique régionale?
}

Paul Alliès, François Baraize, William Genieys, Emmanuel Negrier, Jean-Paul Cheylan, Patrick Brossier, Marie-Thérèse Jourda

\section{To cite this version:}

Paul Alliès, François Baraize, William Genieys, Emmanuel Negrier, Jean-Paul Cheylan, et al.. Les élections régionales du 15 mars 1998 en Languedoc-Roussillon. Une nouvelle singularité politique régionale?. Pôle Sud - Revue de science politique de l'Europe méridionale, 1998, Elections et politiques régionales, 8, pp. 5-40. 10.3406/pole.1998.992 . hal-01393354

\section{HAL Id: hal-01393354 \\ https://hal.science/hal-01393354}

Submitted on 7 Nov 2016

HAL is a multi-disciplinary open access archive for the deposit and dissemination of scientific research documents, whether they are published or not. The documents may come from teaching and research institutions in France or abroad, or from public or private research centers.
L'archive ouverte pluridisciplinaire HAL, est destinée au dépôt et à la diffusion de documents scientifiques de niveau recherche, publiés ou non, émanant des établissements d'enseignement et de recherche français ou étrangers, des laboratoires publics ou privés.

\section{(ㅇ)(1) $\$$}

Distributed under a Creative Commons Attribution - NonCommercial - NoDerivatives 44.0 


\title{
Les élections régionales du 15 mars 1998 en Languedoc-Roussillon Une nouvelle singularité politique régionale?
}

\author{
par Paul Alliès, François Baraize, \\ William Genieys, Emmanuel Négrier, CEPEL-CNRS \\ et Jean-Paul Cheylan, GIP-Reclus Montpellier \\ Cartographie et traitement des données par \\ Patrick Brossier, GIP-Reclus Montpellier \\ et Marie-Thérèse Jourda, CEPEL-CNRS
}

Pôle Sud $N^{\circ} 8$ - mai $1998-p .5$ à 40.

La page du Midi rouge semble définitivement tournée en Languedoc-Roussillon, où la gauche poursuit une mutation d'accompagnement des changements socio-économiques profonds de la région. Mais cette transformation semble sans commune mesure avec le chantier ouvert à droite, où le bloc des forces parlementaires traditionnelles (UDF-RPR) doit désormais compter avec une nouvelle stratégie frontiste, celle de la "main tendue ", dans un rapport de force qui ne lui est plus totalement favorable.

Au soir du 15 mars 1998, aucune majorité absolue ne s'était dégagée des urnes en Languedoc-Roussillon. Pour 67 membres du Conseil Régional, on en comptait 31 à gauche (20 PS, $8 \mathrm{PC}, 1 \mathrm{MDC}$ et $2 \mathrm{PRG}$ ), 22 à droite (10 UDF, 11 RPR et 1 Divers Droite), 13 au Front National, 1 CPNT. Officiellement classée parmi les régions ayant basculé à gauche au soir du scrutin, la Région Languedoc-Roussillon fait pourtant partie des lieux-phares où se discute une éventuelle alliance entre droite et Front National. L'UDF et le RPR y sont cependant en position nettement plus faible que dans les autres régions (Rhône-Alpes, Bourgogne...) où la majorité relative pourrait consacrer un tel tournant. Il faut en effet un accord massif du FN, et une absence presque totale de défection à droite à l'égard de cette stratégie d'alliance pour que Jacques Blanc conserve, à droite, la présidence du Conseil Régional. Faute de majorité, celui-ci avait, en 1992, exclu tout recours au Front National, assuré qu'il était de pouvoir enrôler quelques écologistes pour compléter sa propre position majoritaire, dans un contexte il est vrai très défavorable à la gauche. En 1986, il avait dû à un accord avec le Front National d'être élu contre une gauche majoritaire dans la région. Entre les 15 et 20 mars 1998 (jour de l'élection du Président du Conseil Régional), l'hypothèse d'un accord entre droite et FN pour maintenir J. Blanc à la présidence devient crédible, en dépit du fait que, parmi les nouveaux élus du RPR, certains ont, par leur responsabilité militante, leurs attaches familiales ou les fonctions qu'ils occupent au sein de l'appareil central du parti toutes les raisons de préférer les consignes d'état-major à une alliance régionale "aventureuse". La gauche plurielle, où ne figure aucun représentant écologiste, établit sa propre plate-forme, 


\section{Pôle Sud $N^{\circ} 8$}

autour de G. Frêche et de l'idée d'une présidence collégiale. Le vendredi 20 mars, $\mathrm{J}$. Blanc intègre une partie des exigences du FN (et notamment le "rejet de la dictature culturelle de la gauche") dans son propre texte de candidature. Peu avant midi, J. Blanc est élu au premier tour Président du Conseil Régional avec 35 voix, contre 31 à G. Frêche et 1 au chasseur. Il n'aura pas manqué une voix à l'UDF-RPR-FN pour sceller la nouvelle majorité. Exclu de l'UDF dans la semaine suivante, J. Blanc en appelle à la fondation d'un nouveau parti régional conforme à ses récentes options. Contrairement à d'autres présidents élus dans les mêmes conditions, J. Blanc a, avant le scrutin, fait voter par l'ancienne majorité l'intégralité du budget 1998, ce qui lui évite d'être tout de suite confronté aux conséquences politiques internes de son élection.

Pour analyser les résultats de ces élections, il nous a semblé nécessaire de présenter, de manière synthétique, le contexte socio-économique languedocien. Certaines des tendances électorales profondes relevées plus loin sont pour nous en effet liées à certains aspects du changement social (l'urbanisation, la désindustrialisation, les nouvelles polarisations territoriales...), avant que d'être la traduction immédiate de stratégies partisanes.

Pour autant, ces dernières comptent, et d'abord dans la manière dont l'enjeu régional a été présenté aux électeurs languedociens. C'est pourquoi nous consacrons un chapitre à la campagne électorale ellemême, avant d'analyser, courant par courant, les élections régionales de mars 1998. Nous disposions des données équivalentes pour les deux scrutins antérieurs (1986 et
1992), ainsi que des résultats de toutes les consultations intermédiaires. C'est pourquoi nos analyses font une large place à l'évolution des rapports de force, en général, et par formation politique, avec une cartographie complète en annexe. Outre la description des principales tendances politiques, cet article a également pour ambition d'expliquer pourquoi, dans une région où la gauche est majoritaire, la droite a pu maintenir son leadership régional au prix d'une extension à l'extrême-droite de sa majorité.

\section{Changement social et dynamiques électorales en Languedoc-Roussillon : d'une élection régionale à l'autre}

L'observation du changement social en Languedoc-Roussillon est assez ancienne pour qu'il soit aisé d'en déterminer les tendances les plus récentes '. On ne retiendra ici que celles qui peuvent aider à mieux comprendre les évolutions politiques ressortant des derniers scrutins. Sous cet angle deux lignes forces s'imposent : celle d'une polarisation économique et sociale qui masque la nature fragile de la croissance et celle d'une urbanisation généralisée de l'espace régional.

L'image de la polarisation s'impose avant tout avec le contraste entre la création d'emplois et la progression du chômage. $\mathrm{Au}$ cours des vingt dernières années, la région a vu l'emploi croître de $30 \%$, soit un taux bien supérieur à la moyenne nationale. En même temps le chômage y progressait de $11 \%$ en s'établissant à 4,5\% au-dessus de la moyenne nationale. 


\section{Les élections régionales du 15 mars 1998 en L.-R.}

L'emploi créé est majoritairement localisé entre le sud du Gard et Montpellier. Il l'est par de petites entreprises de moins de dix salariés qui sont fréquemment rachetées. Comme celles-ci forment les $90 \%$ des PME-PMI, le nombre des entreprises de moins de 5 ans est bien plus élevé qu'ailleurs et signale une sérieuse instabilité de l'emploi. Enfin cet emploi reste faiblement industriel $(11,2 \%$ de l'emploi total contre $21 \%$ en France). C'est le secteur des services qui a créé la quasi totalité des emplois nouveaux : le nombre de salariés dans ce secteur a triplé au cours des vingt dernières années, principalement dans la fonction publique (20\% de l'emploi régional, le tiers du tertiaire), le commerce et les services de proximité ( $60 \%$ des emplois régionaux, une croissance trois fois supérieure à la moyenne nationale).

Ces facteurs expliquent la fragilité de ce qui est en expansion en LanguedocRoussillon : le tourisme y est une ressource essentielle ( $12 \%$ du PIB régional soit la plus forte proportion de toutes les régions françaises). 65000 personnes y sont employées, c'est-à-dire presque autant que dans l'agriculture et l'industrie et bien plus que dans le BTP. C'est là que la création d'emploi est la plus forte (plus $68 \%$ contre plus $35 \%$ en France). Mais la moitié de ces emplois sont saisonniers, ce qui aggrave encore un peu plus le sentiment d'instabilité et de précarité, ce qui encourage le travail souterrain dans un secteur où l'obsolescence de l'offre et des équipements est problématique. Le salariat de ce secteur, isolé, faiblement organisé, a tendance à se replier sur les ressources familiales pour organiser son mode de vie. En outre, ce type de croissance ne compense pas les pertes d'emploi dans l'agriculture et l'industrie.

Dans l'agriculture, 22000 salariés ont disparu au cours des vingt dernières années ainsi que 42000 exploitations. La surface moyenne de celles-ci a augmenté de 10 hectares. Une complète réorientation de l'économie viticole s'est accomplie, faisant tendanciellement disparaître l'exploitation familiale et directe. Même la coopération, si importante pour comprendre les comportements communautaires et républicains, est devenue "manageriale".

Dans l'industrie, la vulnérabilité est grande : en dehors de l'agro-alimentaire, le Languedoc-Roussillon ne connaît pas de véritables filières, ni d'orientations organisées vers les marchés extérieurs. Ceci l'empêche de profiter des relances sectorielles. De plus l'industrie régionale est partagée entre secteur formel et informel, moderne et traditionnel. Cette désarticulation la place sous la dépendance de donneurs d'ordre extérieurs, filiales de grands groupes nationaux ou internationaux : $90 \%$ des effectifs industriels en dépendent. Les postes de cadre connaissent la plus forte progression (plus 4,5\% par an depuis 1982), mais une infime minorité de chercheurs (6\% environ) parmi les 8000 que compte la région travaillent pour l'industrie.

Cette vision d'ensemble explique un développement paradoxal où ce qui est ailleurs capitalisé comme ressource (les réseaux familiaux, le municipalisme, le savoir-faire traditionnel, l'histoire locale) est ici facteur de résistance au changement. Le contraste entre la "troisième Italie" (la Toscane ou les Marches) et le Languedoc- 


\section{Pôle Sud $N^{\circ} 8$}

Roussillon est saisissant (Ritaine, 1991; 1989). Les territoires ne sécrètent pas l'entreprise ni de réseaux d'entreprises, sauf peut-être autour de l'agglomération montpelliéraine. L'espace social et politique de la région en porte les traces.

La deuxième ligne-force du changement est en effet celle d'une urbanisation spécifique. Les mutations économiques et démographiques ont intéressé les villes de Montpellier, Sète, Perpignan, Narbonne ainsi qu'une dizaine de communes de 40000 habitants environ. Elles ont accueilli une population venant des autres régions françaises où les pré-retraités et retraités sont nombreux. Les plus actifs de ces nouveaux languedociens ont un rôle important dans les secteurs en forte croissance du tourisme, de la communication et des services en général. Ils sont les plus nombreux (six sur dix) à s'installer dans l'est de l'Hérault où les rejoignent aussi nombre de "natifs" de la région. Mais les villes citées plus haut semblent fixer une population jeune grâce à un essaimage de type universitaire. La population étudiante a doublé en vingt ans dans la région (81 000 étudiants) tout en laissant l'université montpelliéraine loin derrière le dynamisme d'Aix-Marseille, Toulouse ou Barcelone. Tout se passe en effet comme si cette diffusion dans l'espace se payait par une stagnation des pôles forts montpelliérains. Démographiquement, cette fixation d'une population nouvelle et jeune dans les villes principales reproduit la structure fort connue et ancienne du tissu urbain dense du Languedoc-Roussillon. Ce qui y apparaît comme nouveau c'est l'urbanisation en grappe avec la concentration dans les périphéries des villes comme dans les couloirs de communication de phénomènes migratoires è de recompositions sociales difficiles à gérer dans les formes héritées de la période agraire de cette urbanisation. Les problèmes de la ville et de ses modes de vie deviennent ceux de la campagne même quand les avantages de la ville font défaut. Il y a là sans doute, dans ces formes nouvelles d'exclusion et d'anomie locale, une cause du vote frontiste facilement repérable parce que désormais constant dans ces zones. Plus généralement cette urbanisation généralisée, si elle respecte la géographie politique et électorale des principales forces politiques, la perturbe de plus en plus, réduit les écarts, bref banalise toujours un peu plus ce Midi qui était "rouge" il y a encore vingt ans (Alliès, 1995). Ce qui s'est passé depuis les élections régionales de 1986 dans le champ politique le confirme amplement.

\section{La personnalisation de la cam- pagne électorale en Languedoc- Roussillon : des élections sans enjeux "régionaux"?}

Les élections régionales de 1998 ont montré une fois de plus qu'il était difficile ou qu'il n'était pas prioritaire d'axer la campagne sur des enjeux de politique publique régionale. Pour autant, on ne peut dire non plus que celle-ci ait été structurée autour d'enjeux politiques nationaux. Elle s'est en fait constituée autour d'une bataille de leadership personnalisé. Cette réduction de la campagne électorale à un duel d'acteurs se disputant le leadership régional fut affichée 


\section{Les élections régionales du 15 mars 1998 en L.-R.}

avant même les législatives de 1997. Côté socialiste, les deux précédentes campagnes régionales avaient montré la difficulté, dans le cadre d'un scrutin départemental, à construire un leadership régional, et à offrir une figure "présidentiable". Constituées en "comité régional", les cinq fédérations socialistes de la région ont entamé dès le printemps 1996 un travail de mise en synergie et d'acceptation du leadership régional de Georges Frêche, comme figure incontournable d'une victoire aux régionales. Symboliquement, cet adoubement régional aura lieu à Mende, dans les terres lozériennes du Président Jacques Blanc, au mois d'octobre 1996, à grand renfort de couverture de la presse régionale (Midi Libre) et locale (La Lozère Nouvelle). Les législatives inattendues de mars 1997 voient donc un PS régional déjà en ordre de marche électorale. Elles donnent lieu à une très nette victoire des forces politiques de la gauche plurielle, J. Blanc restant le seul député de la coalition UDF-RPR de la région, et par conséquent le leader exclusif de la droite régionale. Le mano a mano peut désormais débuter. Lors d'une conférence de presse en plein été, Blanc prend acte de ce combat singulier, et fustige son principal challenger, le maire de Montpellier. La tactique de "passionalisation" du débat politique régional est désormais partagée par les deux protagonistes, et la presse régionale alimente directement cette lutte des "chefs" en titrant : "BlancFrêche : la guerre des régionales est déclarée" ${ }^{2}$. Cette logique de construction politique de l'adversaire ne va pas sans rappeler les oppositions structurantes qui sont produites par les notables locaux pour mobiliser les électeurs sur le territoire. Le débat sur les politiques régionales est donc occulté au profit d'une "guerre des chefs" entre les deux figures politiques régionales qui utilisent à souhait un répertoire de mobilisation fondé sur la "haine" de l'autre (Birnbaum, 1998 : 48). Ce phénomène n'est pas sans effet pervers sur le déroulement de la campagne pour ce type d'élections. Dans cette perspective, les programmes électoraux sont amenés à tenir un moindre rôle au détriment de la mise en scène de la compétition politique. Ainsi, dès le début du mois de septembre 1997, à Montpellier, lors du lancement de la campagne "officielle", le député maire de Montpellier, entouré des quatre autres têtes de liste socialistes départementales (Roger Adivèze pour l'Aude, Guy Roca pour le Gard, Alain Bertrand pour la Lozère et Jean Carrère pour les Pyrénées-Orientales) dénonce le "bilan catastrophique de la Région" et "l'accord déjà conclu entre Blanc et le $\mathrm{FN}^{\prime}{ }^{3}$. Dès le début de la campagne, la distribution des rôles au sein de l'équipe socialiste reflète sa nouvelle hiérarchie politique. En effet, pendant que le "coordinateur régional" concentre ses attaques générales sur les pratiques politiques du Président sortant, les autres têtes de liste se contentent de critiquer "la politique de Blanc dans leurs départements respectifs" 4 .

La campagne s'effectue sur le terrain autour de cette thématique. Les deux leaders ont sillonné toute la région pour aller à la rencontre des futurs électeurs. Mobilisant des registres politiques fortement personnalisés, leurs approches du territoire furent quelque peu différentes. Le 


\section{Pôle Sud $N^{\circ} 8$}

Président sortant, jouant sur son bilan, mobilise autour de la mise en place formelle des "pays", des élites économiques locales, et consacre un effort plus particulier au département de l'Hérault, où il est venu personnellement défier son challenger. Lors d'une réunion électorale à Montpellier même, il n'hésite pas à exacerber les clivages politiques du département en déclarant à propos de la succession du Président divers gauche du Conseil Général ${ }^{5}$ (qui déclare en janvier ne pas solliciter un nouveau mandat): "Je suis inquiet, Gérard Saumade était quand même un rempart contre la volonté hégémonique de Frêche. Mais il s'en va" ". Ainsi, le candidat Blanc concentre sa communication politique sur la dénonciation des pratiques politiques de son adversaire. Parallèlement, et sur un même registre, le challenger socialiste arpente tout le Languedoc-Roussillon pour faire passer son message. Le leader régional du PS parcourt soixante mille kilomètres, dans une logique cantonale de terrain (un meeting par canton), qu'il complétera d'une centaine de visites d'entreprises ${ }^{7}$. L'apogée de cette logique de personnalisation de la campagne fut l'organisation de deux débats télévisés sur FR3 Languedoc-Roussillon opposant le Président sortant à son challenger. Ce fait constitue une première dans le cadre des élections régionales, aucune manifestation médiatique de ce type n'ayant été organisée lors des précédentes élections régionales. En ne donnant la parole qu'aux deux seuls acteurs ayant réussi à mettre en avant leur probabilité de gouverner l'institution régionale une semaine avant l'échéance électorale, furent exclus du débat médiatique tous les acteurs représentant les autres courants politiques de la région. Les deux derniers mois de la campagne auront d'ailleurs été dominés par le débat sur les alliances majoritaires, et notamment la question de l'alliance entre l'UDF-RPR et le Front National, qui connaîtra en février une première issue juridique (le très médiatique procès en diffamation que $\mathrm{J}$. Blanc intente contre son adversaire socialiste, à propos de l'existence d'un accord écrit entre le Président sortant et le Front National), et son paroxysme lors de la semaine de préparation de l'élection du Président.

On aboutit ainsi à une situation politique extrêmement paradoxale entre un mode de scrutin qui fragmente fortement la représentation politique et la réduction de la campagne à une question de leadership politique personnel. Ce phénomène n'est pas sans effet sur la question problématique de l'institutionnalisation d'une élite politique régionale. Elle n'est pas non plus sans effets sur la visibilité et la légitimité de l'institution, puisque la campagne, ainsi structurée, ne met pas en débat l'action des structures régionales, qu'il s'agisse d'exposer le bilan du sortant, ou d'imposer d'autres dimensions de la politique régionale.

\section{Une participation dans la ligne des autres élections régionales}

Depuis leur première élection au suffrage universel, qui avait vu la désignation des conseils régionaux couplée à celle, également au scrutin proportionnel, de l'Assemblée Nationale, les élections régionales se sont situées dans les fourchettes basses de la participation électorale. Signe que les institutions 


\section{Les élections régionales du 15 mars 1998 en L.-R.}

régionales ne sont pas encore totalement comprises, et que l'enjeu de leur désignation reste faible en termes d'action publique, cette participation réduite semble également refléter le fait que, pour la première fois depuis longtemps, les électeurs ne se sont pas "servi" des élections locales pour adresser un message de sanction au gouvernement, et que celui-ci se trouve dans une conjoncture politique où il n'avait pas réellement besoin de chercher un renfort de ce soutien.

Pour autant, si la moyenne nationale reste faible $(40,2 \%)$, la participation régionale se situe trois points au-dessus, avec de fortes variations par département. La personnalisation de l'enjeu électoral autour des deux figures du leadership régional a certainement contribué à cette mobilisation différentielle. On est ici dans un scrutin où l'enjeu local est fort et largement identifié (Parodi, 1992, p. 281). Ce qui signifie également que la conquête d'un exécutif régional devient un enjeu local, ce qui n'était pas le cas jusquelà. La structure de l'offre politique témoigne de cet enjeu. Il n'y a pas de localisme des candidatures : seuls les électeurs des PyrénéesOrientales se voient proposer une liste régionaliste, dont le score est d'ailleurs en perte de vitesse. La seule spécificité de l'offre régionale est constituée par la présence des listes de chasseurs (CPNT et Chasse-Ruralité). Cette limitation de l'offre politique, qui est en partie la conséquence de la personnalisation de la campagne autour des deux "présidentiables", dans un système d'alliance à la fois prédéfini et pré-dénoncé, contribue à limiter la participation. Les élections régionales sont entrées dans une logique majoritaire.

Il n'est par conséquent pas étonnant que l'indifférence à l'enjeu régional marque encore un pas par rapport aux échéances antérieures, la participation se situant en 1998 deux points en dessous de celle de 1992. Enfin, les votes blancs et nuls, indicateurs de ce refus de l'offre électorale (Bon, Cheylan, 1988, p. 312), sont à leur taux maximum, à près de $5 \%$ des votants.

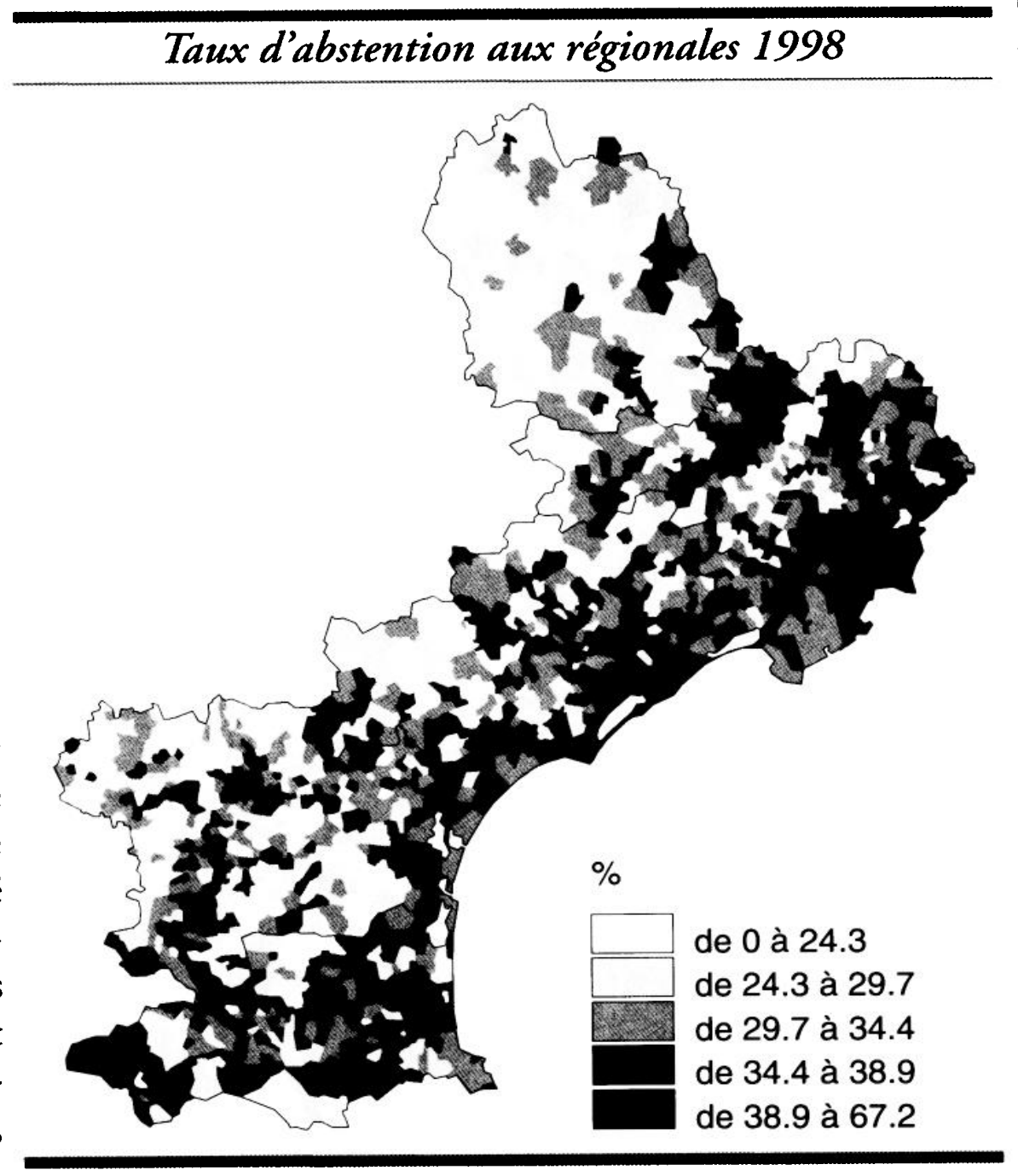




\section{Pôle Sud $N^{\circ} 8$}

Au-delà de ces considérations générales, la localisation et les comparaisons entre les scrutins précédents permettent de donner une carte précise des comportements abstentionnistes.

\section{Une abstention largement différenciée sur le territoire}

On sait les différences traditionnelles de participation entre zones rurales et urbaines. L'abstention de 1998 obéit à ces règles générales, et la plupart des agglomérations importantes de la région connaissent des taux de participation plus faibles que le reste du territoire : Nîmes, Alès, Montpellier, Béziers, Sète, Narbonne, Carcassonne, Perpignan. La Lozère reste, quant à elle, le département le plus participationniste de France.

Pourtant, plus qu'un problème d'urbanité ou de ruralité, ces zones de forte abstention peuvent être repérées à l'aide d'autres variables.

\section{Une complémentarité avec le vote FN}

Plus que jamais, les zones de forte abstention dessinent également les zones où le FN réalise ses meilleurs scores. Outre les agglomérations urbaines, on retrouvera ainsi les zones en reconversion industrielle. Tout le bassin nîmois, jusqu'à la Petite Camargue, et plus généralement tout l'Est gardois. De même, l'ensemble du bassin d'Alès, et d'importants pics autour du Vigan. Dans l'Hérault, le littoral, et de façon très prononcée, toute la zone Frontigan-Mèze, et l'ensemble des zones urbaines biterroises (au contraire du district montpelliérain où seule la ville-centre connaît une importante abstention). Dans l'Aude, on retrouvera là le Narbonnais, plus globalement le littoral, et aussi la région de Limoux et les banlieues de Carcassonne. Dans les Pyrénées-Orientales, toujours le littoral, et la grande agglomération perpignanaise.

Dans cette complémentarité avec le vote $\mathrm{FN}$, on va trouver également les nouvelles zones périurbaines constituées le long de vallées de communication, ainsi que les zones industrielles en reconversion : la vallée du Perthus, Arles-sur-Tech, LézignanCorbières, l'axe Frontignan-Lodève de la RN112, ainsi que l'axe Béziers-Lodève, et enfin la zone de St-Ambroix dans le Gard. Si l'on y ajoute la carte de l'extrêmegauche, on se trouve face à une très grande complémentarité des comportements protestataires.

\section{Une abstention des marches et des périphéries régionales}

Le deuxième enseignement des cartes de l'abstention est en effet la précision avec laquelle elle dessine les territoires qui n'entrent pas dans les logiques centripètes d'intégration régionale et/ou départementales. On trouve la plus forte zone d'abstention régionale dans la partie occidentale des Pyrénées-Orientales : toute la région de Saillagouse, Mont-Louis, Olette, qui revendique son identité catalane sans qu'un mouvement régionaliste et/ou nationaliste ne puisse capitaliser ce sentiment. Plus haut, au sud-Ouest audois, le triangle Axat, Belcaire, Quillan. Entre l'Aude et l'Hérault, la partie occidentale du Minervois, d'Azille à Rieusec. Dans le Gard, les deux zones montagneuses frontalières de la Lozère 


\section{Les élections régionales du 15 mars 1998 en L.-R.}

(Trèves au Sud, Genolhac au Nord), et enfin la seule véritable zone abstentionniste de Lozère : la vallée de Florac-Barre des Cévennes. De façon significative, on retrouve là toutes les zones situées au sudouest des quatre départements littoraux.

\section{Des cantons en vacances}

Au-delà des deux premiers groupes cohérents, on retrouve ensuite ces cantons qui ont cumulé l'absence de renouvellement de leur conseiller général, et l'absence de toute personnalité locale dans les listes régionales, et dont les électeurs ont eu plus de mal à se mobiliser (avec des pics d'abstention à $45 \%$ dans certains de ces cantons ruraux). On sait l'effet d'entraînement certain de ces deux critères. L'abstention est en moyenne de 1,5 supérieure dans les cantons non-renouvelables. Au contraire de ce que certains commentateurs avaient pu prévoir, les régionales ne s'imposent pas comme le scrutin urbain qui serait le pendant des scrutins locaux, et la faiblesse de la nationalisation des votes se confirme dans ce différentiel. Lélection régionale ne "tire" pas les consultations locales. Au contraire, les élections cantonales permettent, là où elles ont lieu, une meilleure participation au scrutin régional, même si cette dernière reste globalement en dessous (de 1 à 2 points) de la consultation départementale.

\section{Qui perd des électeurs?}

Dernier élément permettant de cerner l'abstention, l'analyse des pertes en voix peut éclairer la structure partisane de ces abstentions. Il est difficile pourtant d'établir cette comparaison sur les deux élections régionales, tant les scrutins sont à la fois distants et différents dans leur conjoncture. Mais la limitation de l'offre politique à une offre classique, la personnalisation des enjeux régionaux, la constitution d'une logique de blocs majoritaires clairement identifiés, autorisent à donner un coup de sonde sur les dernières échéances, très rapprochées dans le temps, des législatives de juin 1997.

Entre le ler tour des législatives 1997 et le tour unique des régionales, ce sont près de 120000 électeurs qui manquent à l'appel sur l'ensemble de la région, et 90000 suffrages exprimés, alors que, durant l'année écoulée, la région a gagné plus de 40000 électeurs $^{8}$. La perte de suffrages exprimés est donc de l'ordre de $9 \%$. La présence des chasseurs rend difficilement lisible ces déplacements, car il n'est pas acquis qu'ils prennent toutes leurs voix dans le réservoir de la droite parlementaire. Globalement, le bloc de la gauche plurielle et des écologistes perd 80000 voix, soit $16 \%$ de leur électorat de 1997, le Front National perd également $16 \%$ de son électorat, soit 32000 voix, la droite parlementaire se situant légèrement au-dessous de la moyenne régionale, en perdant 24000 voix, soit $8 \%$ de leur électorat de 1997 . Les formations non présentes aux élections législatives s'octroient le solde positif : 47000 voix pour les chasseurs et 2000 pour les régionalistes catalans. Hormis ces formations "conjoncturelles", seuls trois partis améliorent leur implantation au sein du potentiel électoral global : le Parti Socialiste $(+1 \%$ des inscrits), les Verts $(+1,06 \%$ des inscrits) et l'extrême-gauche $(+0,24 \%$ des inscrits). Nous verrons plus bas que le maintien des deux premières formations est largement dû à des recompositions internes à leur propre champ (réintégration de votes divers gauches et divers écologistes). C'est donc l'ensemble des 


\section{Pôle Sud $N^{\circ} 8$}

formations "de gouvernement" qui perd des électeurs. On est bien dans une conjoncture de désaffection de l'enjeu régional.

\section{Une droite régionale stagnante?}

Si lors de nos analyses comparées des élections régionales de 1986 et de 1992 nous avions montré un affaiblissement général de l'électorat de droite, force est de constater que pour les élections de 1998, cette dernière maintient un statu quo relatif.

En effet, la mise en perspective des deux premiers scrutins régionaux montrait que la droite en Languedoc-Roussillon avait subi la concurrence directe de la montée électorale du front national. La difficulté qu'avaient rencontrée les listes uniques de la droite régionale et le surinvestissement du Président Jacques Blanc dans son fief lozérien s'étaient traduits par un recul certain de son électorat. L'effet de limitation de l'offre électorale était pour nous un des éléments avancés pour interpréter ce recul. Seul le département de l'Hérault, où la formation d'une liste dissidente menée par Jean Pierre Grand n'avait pu être évitée, voyait la droite maintenir ses positions électorales. À cette même échéance électorale, la droite s'affermissait sur ces zones de forces, tel son territoire de prédilection, la Lozère. On avait noté toutefois que dans le sud de ce département où le clivage catholicisme/protestantisme perdure dans les cantons de St Germain de Calberre, de Barre des Cévennes et de Pont de Montvert en prolongeant le nord du Gard protestant, la liste de Jacques Blanc ne dépassait pas $35 \%$. Les élections régionales 1998 ont confirmé cette évolution sur ce même territoire où la liste PS remporte un siège en obtenant $19,82 \%$ des voix. On note cependant que contrairement à 1992, Jacques Blanc ne se présentait pas comme tête de liste de la droite. Une liste RPR dissidente menée par Francis Saint Léger a obtenu $11,52 \%$ des suffrages exprimés tandis qu'une autre liste dissidente menée par un 


\section{Les élections régionales du 15 mars 1998 en L.-R.}

anonyme homonyme du Président sortant, Daniel Léonard Blanc totalise $0,75 \%$ (soit 315 voix). Sans surévaluer cette anecdote qui ressemble à un "vaudeville" électoral, il semble que le leadership politique du Président de la Région soit contesté sur la terre de prédilection des anciens "maîtres de granit". La liste Hugon, UDF-RPR régresse sur les terres mêmes de prédilection du Président Blanc : l'Aubrac l'ouest lozérien (jusqu’à la Canourgue).

Évolution des résultats électoraux de la droite parlementaire en L.-R, 1986, 1992, 1998

\begin{tabular}{|c|c|c|c|c|c|}
\hline DEPART. & AUDE & GARD & HÉRAULT & LOZERE & P-O \\
\hline \multicolumn{6}{|c|}{ Régionales 1986} \\
\hline$\%$ & 28,56 & 37,25 & 36,28 & 52,72 & 37,50 \\
\hline Nb Sièges & 4 & 7 & 9 & 2 & 4 \\
\hline \multicolumn{6}{|c|}{ Régionales 1992} \\
\hline$\%$ & 31,66 & 24,89 & 31,50 & 63,29 & 28,01 \\
\hline Nb Sièges & 4 & 5 & 8 & 3 & 4 \\
\hline \multicolumn{6}{|c|}{ Régionales 1998} \\
\hline$\%$ & 30,60 & 28,90 & 28,00 & 63,71 & 27,57 \\
\hline Nb Sièges & 3 & 5 & 8 & 2 & 4 \\
\hline
\end{tabular}

Dans l'Aude, la droite s'est présentée divisée autour de deux listes : la première, celle dirigée par "l'héritière" du notable de la cité de Carcassonne, Isabelle Chesa, et la seconde, menée par Alain Madalle et Daniel Arrata, deux ex-députés de la vague bleue de 1993 qui contestent à la fois le leadership départemental des barons urbains (Chesa à Carcassonne, Mouly à Narbonne), la transmission d'héritage à la fille Chesa et le positionnement de cette dernière dans l'accord à venir avec le Front National. La mise en forme définitive des listes de droite avec des épisodes allant de la fusion pour finir par le retrait de l'investiture RPR à la liste Chesa n'a eu pour effet d'affaiblir que faiblement la droite (diffé- rentiel 92/98: $1 \%$ ). On note toutefois que Jacques Blanc n'a cessé de soutenir la liste Chesa qui en retour lui assurera un soutien indéfectible lors de l'élection à la présidence, alors que Philippe Seguin jouait au "nonsoutien participatif" refusant le label RPR aux deux listes, mais venant soutenir Daniel Arrata dans une campagne cantonale.

Dans le département du Gard, la liste Roustan affichant la bannière de la majorité régionale malgré deux listes dissidentes concurrentes progresse de $4 \%$ par rapport à la dernière échéance régionale. Ce résultat est assez surprenant dans la mesure où dans ce département la droite a perdu depuis la ville de Nîmes, et que le processus de rénovation entamé autour du maire d'Alès demeure relatif, comme le montrent les candidatures émanant de RPR dissidents tels que Max Romanet, ou encore Joëlle Pijot (liste femmes). Sans doute, la recomposition chaotique de l'électorat de l'ancien Président du Conseil Général, MRG, puis divers gauche, puis RPR puis France Unie, a-t-elle permis de renforcer une partie de son électorat. La droite en profite et renforce son implantation dans la plaine du Gard (cartes 1 et 2), à l'exception notable des zones où le FN progresse lui-même.

Le département de l'Hérault est la circonscription électorale sur laquelle les deux prétendants à la présidence de Région s'affrontaient. Jacques Blanc avait décidé de se présenter en tête de liste sur ce territoire pour s'opposer directement au maire de Montpellier. Ce département était une sorte de test pour la droite régionale dans la mesure où il y est pourvu le plus grand 


\section{Pôle Sud $N^{\circ} 8$}

nombre de sièges (24). La campagne électorale se résuma à la mise scène par la presse locale et les médias d'un "combat des chefs" censé mobiliser la population locale. Cette stratégie partagée par les deux leaders n’a pas réussi à enrayer la dynamique abstentionniste. Ainsi, le Président sortant n’a pas pu faire remonter le poids électoral de la droite héraultaise en totalisant $28 \%$, soit $3,5 \%$ de moins qu'en 1992. Pourtant, Jacques Blanc avait cette fois réussi à éviter toute liste dissidente à droite en réduisant les velléités de l'ex-député RPR, Bernard Serrou. Cependant, Jacques Blanc peut se prévaloir, d'une part, d'avoir devancé son challenger socialiste de 207 voix $(0,6 \%)$ et d'autre part, de pouvoir prétendre avoir fait reculer le vote $\mathrm{FN}$ de $1 \%$ !

Enfin dans les P.-O. la liste conduite par Arlette Franco, maire de Canet-enRoussillon se maintient dans une position de quasi statu quo par rapport à 1992 $(-0,4 \%)$. On note toutefois que la droite dans ce département est aussi en pleine restructuration. En effet, le pouvoir notabiliaire de la droite construit autour du conseil général des P.O. vient d'être mis à mal par la perte de ce dernier au profit des forces de gauche. Cette défaite de la droite républicaine UDF-RPR pourrait accélérer la recomposition de la droite perpignanaise à l'œuvre depuis les dernières élections municipales et l'élection de Jean-Paul Alduy à la tête de la municipalité, contre les candidats investis nationalement, et notamment les dauphins de son propre père, Paul Alduy.

En guise de conclusion sur l'évolution du comportement de la droite aux élections régionales, on note le paradoxe suivant : malgré la stabilisation relative de l'électorat de droite, cette force politique perd deux sièges (24 en 1992 contre 22 en 1998). Par ailleurs, le Président sortant J. Blanc a encore réussi à imposer son leadership politique sur le territoire régional. Tout d'abord, il a structuré des listes départementales regroupées sur la dénomination "majorité régionale" autour d'acteurs lui vouant une "fidélité politique" à toute épreuve, faisant comme si l'alliance de fait avec les voix du FN lors de son élection à la présidence ne devait pas entraîner des défaillances dans son camp. On doit aussi remarquer que le processus de "dénotabilisation" des listes n'a pas entraîné un affaiblissement électoral comme on aurait pu le penser. Enfin, si de façon générale l'électorat de droite se maintient, on note que les bastions relatifs de l'Aude et des PyrénéesOrientales de l'intérieur, comme ceux plus récents de la basse vallée de l'Hérault et du nord montpelliérain ne s'affirment que peu. L'ensemble du littoral, à l'exception du Narbonnais, montre une faiblesse inhabituelle. En revanche le Gard intérieur, du Rhône à Anduze, confirme son glissement vers la droite.

\section{La recomposition de la gauche}

Les élections régionales de 1992 ainsi que les législatives de 1993 avaient marqué pour la gauche en Languedoc-Roussillon, la fin d'un double cycle : le cycle long des implantations territoriales et culturelles et le cycle plus court du congrès d'Epinay du Parti Socialiste.

Concernant la carte de la gauche, ces élections avaient révélé le déclin linéaire du 


\section{Les élections régionales du 15 mars 1998 en L.-R.}

socialisme viticole dans l'Aude comme dans le Biterrois, la régression socialiste quasi absolue en Roussillon, le maintien d'une influence significative dans les villes aussi bien pour le PS dans celles qu'il avait conquises depuis 1977 que pour le PC dans celles de sa vieille tradition municipaliste (Alès, Nîmes, Sète). La gauche semblait ainsi pouvoir résister grâce à un repli sur ses bastions, ceux du socialisme républicain, du communisme municipal et du modèle technopolitain. Politiquement cette carte ne semblait pas contenir de dynamique propre.

Concernant la structuration politique de la gauche, cette période était celle d'un recul du PS plus fort dans la région qu'au niveau national (moins $21 \%$ contre $16 \%$ ) au profit d'une abstention galopante et des écologistes qui réussissaient une vraie percée ( $13 \%$ aux régionales et $9,11 \%$ aux législatives). Le PC reculait lui aussi mais moins et moins vite que nationalement. Aucun leadership ni partisan ni personnel ne semblait pouvoir s'imposer. La crise interne du PS en était un facteur principal, illustrée tout autant par les rivalités entre le maire de Montpellier et le Président du Conseil Général de l'Hérault que par les oppositions entre fédérations (fabiusiennes dans l'Aude, jospinistes dans l'Hérault) et aussi par des affaires paralysant son personnel notabiliaire comme dans le Gard. Ce parti qui n'avait jamais complètement réalisé son congrès d'Epinay localement dévoilait alors au grand jour ses "sous-cultures", plus radicales, républicaines et francmaçonnes que social-démocrates.

Les élections de 1995 ont sans doute ouvert un nouveau cycle. Aux présidentielles la gauche dépassait la droite de plus de deux points et retrouvait des scores supérieurs à ceux de la fin des "années Mitterrand". Aux municipales, elle confortait ses positions ou les reconquérait dans les anciens bastions communistes. Le retour à une stratégie unitaire fondée prioritairement sur le PS et le PC s'avérait payant. Les législatives de 97 allaient le démontrer partout : la candidature unique dès le $1^{\text {er }}$ tour du communiste Villa dans les Pyrénées-Orientales et son élection au $2^{e}$ tour dans une circonscription difficile, en fut le symbole. De manière générale les candidats du PC n'eurent qu'à se féliciter d'un retour à l'alliance privilégiée avec le PS aussi bien à La Grand-Combe, qu'à Nîmes ou à Sète. Contrairement aux Verts, ils ont enregistré de nettes progressions dans ces législatives en capitalisant les dynamiques d'union des municipales. Mais c'est le PS qui mit le mieux à profit ces élections pour rebâtir son influence. L'exemple le plus net est celui de l'Aude où ce parti fit le plus grand bond en avant de la région : c'est là aussi où il avait le plus renouvelé et rajeunit ses candidats tels Jean-Claude Pérez (33 ans) élu à Carcassonne et Jacques Bascou (44 ans) à Narbonne. Ces cas ne sont pas isolés : Alain Fabre-Pujol (33 ans) élu à Beaucaire, Christian Bourquin (42) à Perpignan, Jean Codognès (44) à Rivesaltes, Damien Alary (46) au Vigan en témoignent. La plupart d'entre eux sont aussi hommes d'appareil et réalisent une osmose plus forte qu'auparavant entre le parti et les électeurs. Cette dimension a d'ailleurs joué à plein pour la préparation des élections régionales. Le Languedoc-Roussillon fut une des rares régions où la structure du "Comité régional" prévue par les statuts fut mise en place et fonctionna réellement. Coordination des 


\section{Pôle Sud $N^{\circ} 8$}

cinq fédérations départementales, elle fut non seulement une instance apportant un soutien logistique effectivement régionalisé à Georges Frêche mais aussi un lieu de regroupement des cadres intermédiaires du parti. C'est ainsi que le PS a profité d'un reflux du vote protestataire anti-européen tel qu'indiqué par le non au référendum sur le traité de Maastricht en 1992 ou par les listes faisant de ce refus leur thème central aux européennes de 1994 : le PS progressait en 97, au détriment du PC, dans des zones où les pourcentages de ces votes étaient exceptionnellement élevés (Biterrois, vallée de l'Hérault, Carcassonne et Narbonne). Ceci est de nature à éclairer le renouvellement de l'offre politique que les élections régionales allaient confirmer : celle d'une gauche plurielle mais polarisée fortement par un socialisme urbain et moderniste, attractif pour les tranches d'âge jeunes et pour les couches moyennes salariées.

Les écologistes semblent faire les frais d'une telle recomposition : les électeurs qu'ils avaient gagnés en nombre au PS en 1992 et 1993 n'ont pas confirmé leur soutien, les nouveaux préférant le vote utile pour le PS. Mais le PC n'est pas non plus véritablement gagnant : par rapport aux régionales de 1992, il semble se maintenir mais sans pouvoir rattraper la régression enregistrée à l'époque. Ainsi dans le Gard il
Vote PC, PS et divers gauche aux régionales 1998

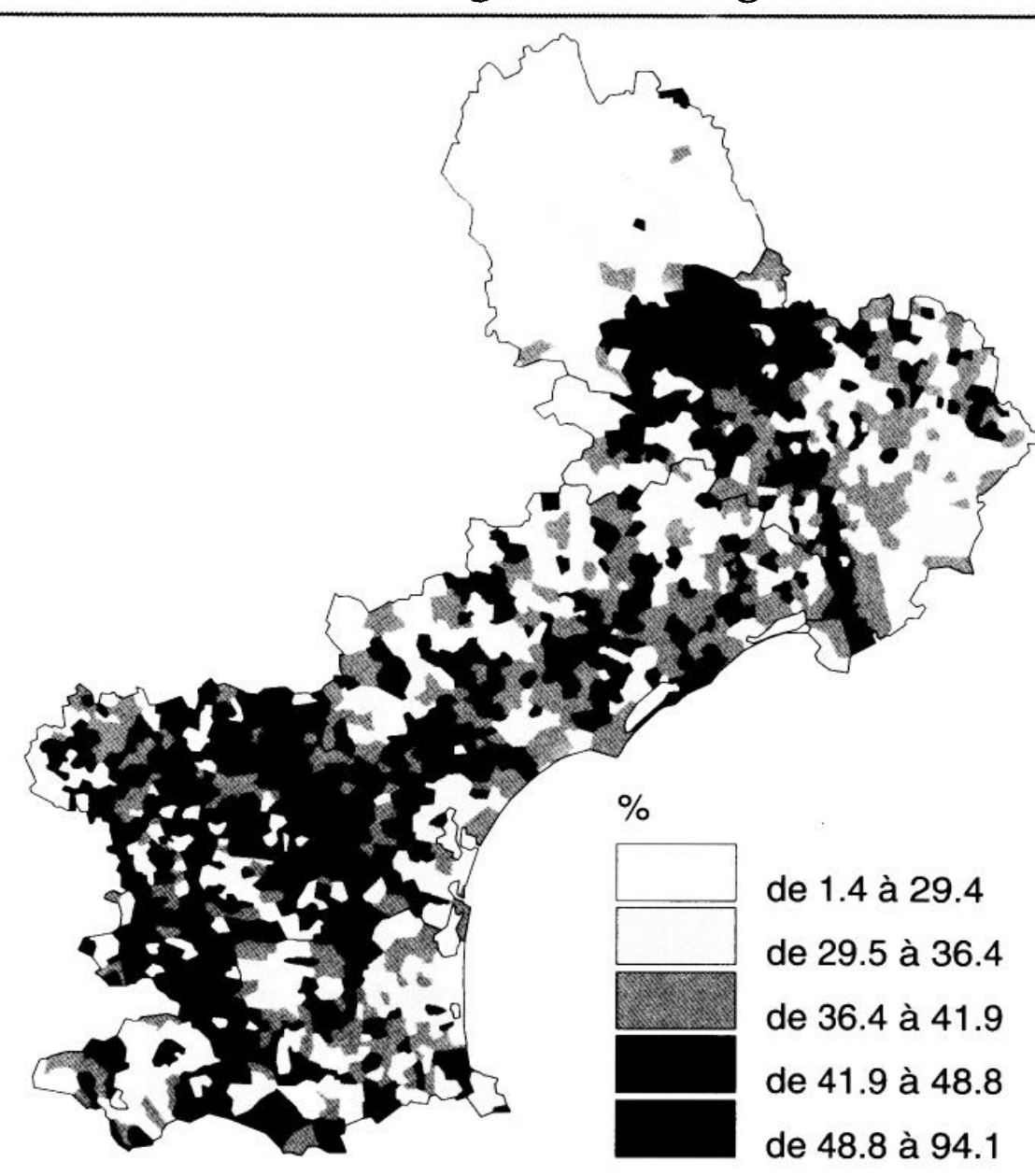

gagne $1,7 \%$ en 1998 mais il avait perdu plus de $6 \%$ en 1992. Dans l'Hérault il ne recule que de $0,3 \%$ en 1998 mais il avait reculé de $3 \%$ en 1992 . Surtout, il se laisse supplanter dans ces deux départements par la liste socialiste dans ses bastions municipaux. La cause n'en est pas le score de l'extrême-gauche qui reste très modeste par rapport au reste de la France $(2,75 \%$ contre 4,38 ) et qui n'est pas corrélé avec le vote communiste. Le cas du Languedoc-Roussillon permet ainsi de confirmer la tendance nationale : la stratégie de la liste unique n'a pas été un moyen sûr pour gagner des voix, bien au contraire. Dans les départe- 


\section{Les élections régionales du 15 mars 1998 en L.-R.}

ments où les comparaisons sont possibles la gauche a plus reculé qu'elle n'a progressé vis-à-vis des élections législatives de 1997 quand elle présentait une liste unique (cartes 3 et 4). Dans la région, c'est dans l'Hérault où chacune des composantes allaient au combat séparément que l'écart en voix avec les législatives est le moindre $(-0,3 \%)$ et où la progression sur les régionales de 1992 est la plus élevée (plus $15 \%$ ); dans le Gard la régression est de $3 \%$. Mais dans l'Aude où PS et PC faisaient liste unique la régression est de $7,3 \%$ et dans les P.O. de $2,4 \%$. Les quelques centaines de voix qui séparèrent le score des Verts de la barre des $5 \%$ de suffrages exprimés dans l'Hérault comme dans le Gard ont sans doute enlevé un siège à la gauche régionale. Mais les analyses et projections sur le calcul des restes à la plus forte moyenne ne sauraient contredire ce qui ressort du comportement basique de l'électorat.

Au total, le nouveau cycle ouvert en 1995 et que ces élections régionales distinguent prend appui sur les zones de force historiques de la gauche : Cévennes et piémonts cévenol, audois, pyrénéen. Il marque un renouveau dans le tissu urbain du centre héraultais et dans l'agglomération montpelliéraine. La zone littorale (sauf Sète-Frontignan et la côte Vermeille), peuplée d'électeurs absentéistes, retraités et saisonniers échappe de plus en plus à son influence. De même des secteurs comme les hauteurs de la $4^{e}$ circonscription législative de l'Hérault aux confins du Gard, autrefois représentés par la gauche, semble lui faire défaut. Il sera donc intéressant de suivre les effets politiques de la contradiction institutionnelle que la gauche va devoir gérer : surmobilisée autour de la personnalité de Georges Frêche pour diriger la Région, elle se retrouve notoirement renforcée à la tête des départements, soit qu'elle y ait gagné de nouveaux sièges là ou elle était déjà majoritaire (mais souvent au bénéfice des affiliations partisanes comme dans l'Hérault et le Gard), soit qu'elle ait conquis des majorités nouvelles comme dans les Pyrénées-Orientales. Si elle se confirme, la coordination interdépartementale des politiques de gauche dans quatre conseils généraux sur cinq ${ }^{9}$, plus proche des intérêts des partis comme des groupes d'intérêts peut être un facteur d'homogénéisation relative de son influence dans la région. L'isolement d'un exécutif régional sous l'influence du Front National, tant vis-à-vis de Paris que de Bruxelles et des régions européennes partenaires pourraient avoir ce résultat paradoxal de rabattre la gauche régionaliste sur l'institution départementale et sur un meilleur enracinement, localiste et cantonal.

\begin{tabular}{lccccccc}
\hline \multicolumn{7}{c}{ Evolution des scores de la gauche en $L .-R$ en $\%$} \\
\hline & AUDE & GARD HÉRAULT & LOZERE & P.O. & RÉGION \\
Régionales 1992 & 41,0 & 40,4 & 28,0 & 23,7 & 27,4 & 33,1 \\
Législatives 1993 & 42,4 & 38,0 & 35,7 & 20,6 & 31,7 & 26,0 \\
Européennes 1994 & 46,8 & 40,9 & 39,5 & 28,5 & 38,3 & 40,4 \\
Présidentielle 1995 & 47,7 & 39,5 & 39,6 & 31,7 & 38,6 & 40,3 \\
Législatives 1997 & 50,4 & 41,4 & 43,3 & 33,8 & 41,2 & 43,2 \\
Régionales 1998 & 43,1 & 38,3 & 43,0 & 27,5 & 38,8 & 40,4 \\
\hline
\end{tabular}




\section{Pôle Sud $N^{\circ} 8$}

\section{Chasseurs et Écologistes : la marge éclatée?} est en présence de deux représentations, plus antagoniques que complémentaires, de la nature comme objet politique. Ce qui marque ce scrutin régional, c'est la fragmentation partielle de ces deux camps. Les listes de chasseurs se sont parfois présentées en ordre dispersé, comme dans l'Hérault, où le sortant, $M$. Cabannes, devait affronter le Président de la Fédération Départementale. Les écologistes ont eu maille à partir avec la dynamique de la i gauche plurielle, à laquelle ils ne se sont pas
Avec les listes chasseurs et écologistes, on

associés, dans presque tous les départements, en présentant des listes séparées.

\section{Chasseurs et protecteurs?}

Pour ces courants récemment présents sur la scène politique les effets de liste demeurent très importants, avec un affaiblissement général dans le Gard, et un renforcement dans l'Aude, où les scores de 1992 étaient cependant très faibles. Malgré la concurrence interne, les résultats sont quasi inchangés dans l'Hérault (carte 5 en annexe). Au-delà de ces variations, le courant politique s'exprime par une structuration régionale qui s'enracine. Les mêmes zones de force et de faiblesse qu'en 1992 et 1989

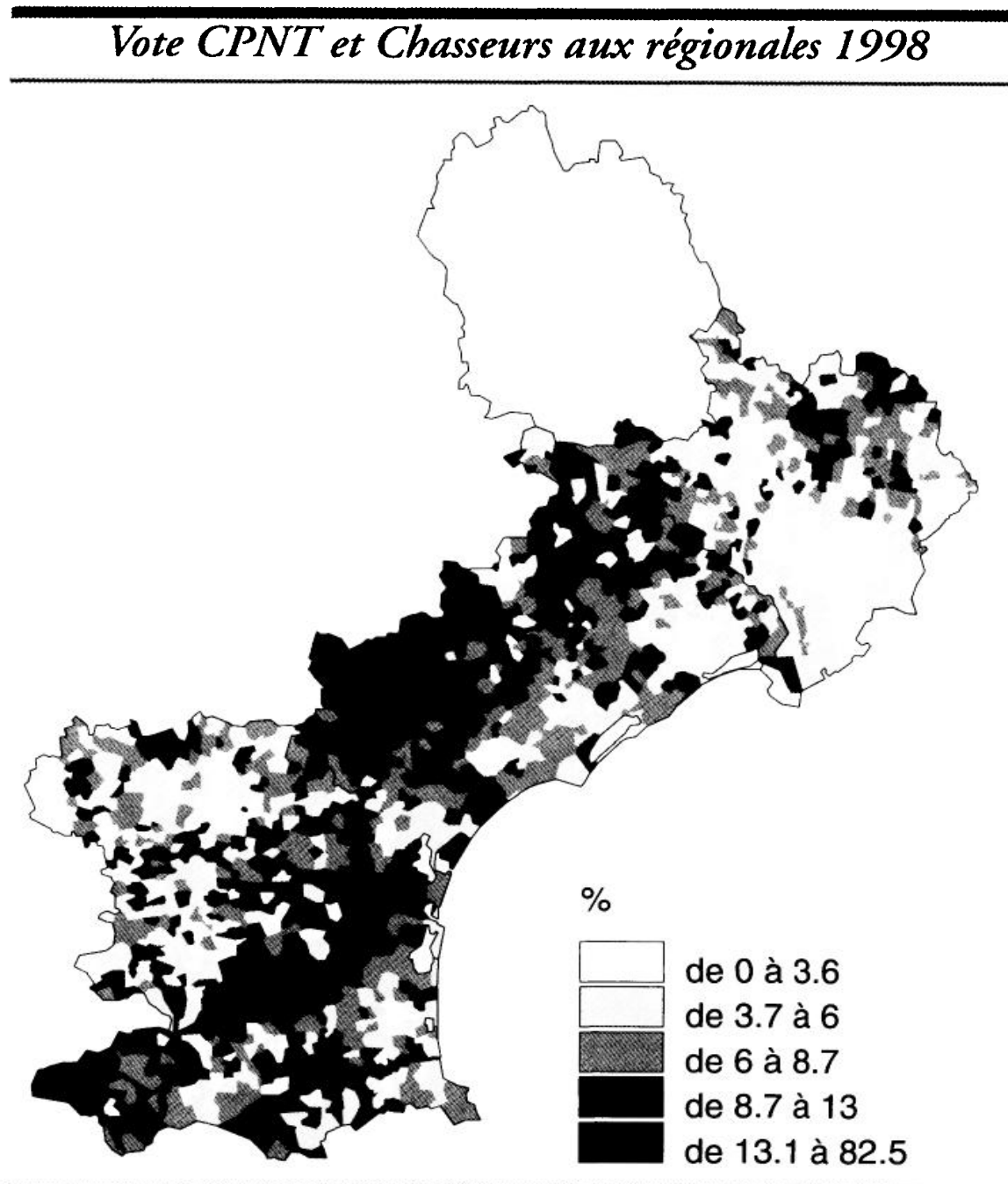
apparaissent, zones qu'il partage souvent avec les diverses expressions écologistes, même si l'on peut faire l'hypothèse que les deux courants reposent sur des strates sociales quasiment disjointes et souvent antagonistes. En Thomières, Minervois, Haut Biterrois, Espinousse et Escandorgue, soit presque tout le quart nord-ouest du département de l'Hérault, les chasseurs obtiennent en général plus de $13 \%$ des suffrages exprimés. Dans les Cévennes gardoises et dans la frange nord-est de l'Hérault, de même que sur les causses, apparaissent également des espaces de force. Les résultats y sont plus contrastés, moins homogènes qu'en 1992, surtout dans le Gard qui consti- 


\section{Les élections régionales du 15 mars 1998 en L. $R$.}

tue, pour ce scrutin, un espace de faiblesse. Dans les Pyrénées-Orientales, la répartition demeure très proche de celle de 1992, avec des forces réparties dans les espaces convoités : Capcir et Cerdagne, versant nord du Canigou et sillon des Fenouillèdes. Ces zones d'influence sont toutes liées à des régions de pratique intensive de la chasse, et surtout de la chasse terrestre. Il s'agit donc presque toujours des régions rurales les plus dépeuplées. En contrepartie, les villes n'apportent que peu de voix à ce courant. Parmi les espaces de faible densité humaine, les chasseurs prennent particulièrement place, au plan électoral, dans ceux qui sont actuellement soumis à des procédures de protection de l'environnement. Il s'agit de Parcs Nationaux (Cévennes) ou Régionaux (Haut Languedoc), même si, dans ces deux cas, la chasse demeure autorisée, le Parc des Cévennes étant l'un des rares à l'autoriser en zone centrale. L'évolution des votes favorables aux chasseurs renforce cette interprétation : il émerge dans les régions dans lesquelles des dossiers de Parcs Régionaux sont en cours d'instruction, comme le sud-est des Corbières. Leur progrès dans les PyrénéesOrientales met surtout en évidence les Fenouillèdes et les communes du Canigou. Les quelques positions littorales qu'ils avaient acquises en 1989 et 1992 dans les zones humides de chasse au gibier d'eau, en Petite Camargue en particulier, n'apparaissent plus comme des pôles importants. Seules les communes de Gruissan, Vendres et Valras se distinguent dans un espace de faiblesse générale.

Les tentatives de ralliement des courants identitaires locaux ("la Bouvine"), dans l'Hérault et le Gard en particulier, semblent avoir échoué. Ni le lunellois, ni l'outre-
Vidourle (son équivalent des cantons de Vauvert, Saint-Gilles et Marguerittes) n'apportent de soutien à ces listes, même lorsque ces pratiques se superposent à des zones de chasse au gibier d'eau, autour des étangs. Les deux seuls "courants" en progression régulière dans ces espaces sont le Front National... et l'abstention. La fluidité entre les divers comportements protestataires pourrait laisser penser qu'ont lieu de nombreux transferts de voix, souvent circonstanciels ou contingents à des personnalités locales.

\section{D'un écologisme aux autres}

L'écologie, en tant qu'expression politique, demeure un courant fort peu homogène et dont la permanence est difficilement cernable. Les reconfigurations institutionnelles, comme les transferts de personnalités, les diverses alliances, explicites ou non, devraient donc conduire à des résultats assez difficilement interprétables sinon erratiques. Or il n'en est rien, ceux-ci sont dans l'ensemble très faibles dans tous les départements. Les listes de la gauche plurielle semblent bien avoir rassemblé une grande partie du courant. La répartition régionale de l'écologie se confirme donc (carte 6 en annexe), avec ses meilleurs résultats dans des espaces ruraux de l'intérieur, assez souvent ceux qui attirent le plus les populations néo-rurales : Cévennes, Plateau de Lussan, Causses, Thomières et Minervois, Corbières sud et Haute vallée de l'Aude. L'écologie affiche une faiblesse régulière dans les grandes villes et les implantations péri-urbaines, sauf au nord de Montpellier où elle rencontre les couches intellectuelles de la capitale régionale. 


\section{Pôle Sud $N^{\circ} 8$}

\section{Vote Verts et divers-écologistes aux régionales 1998}

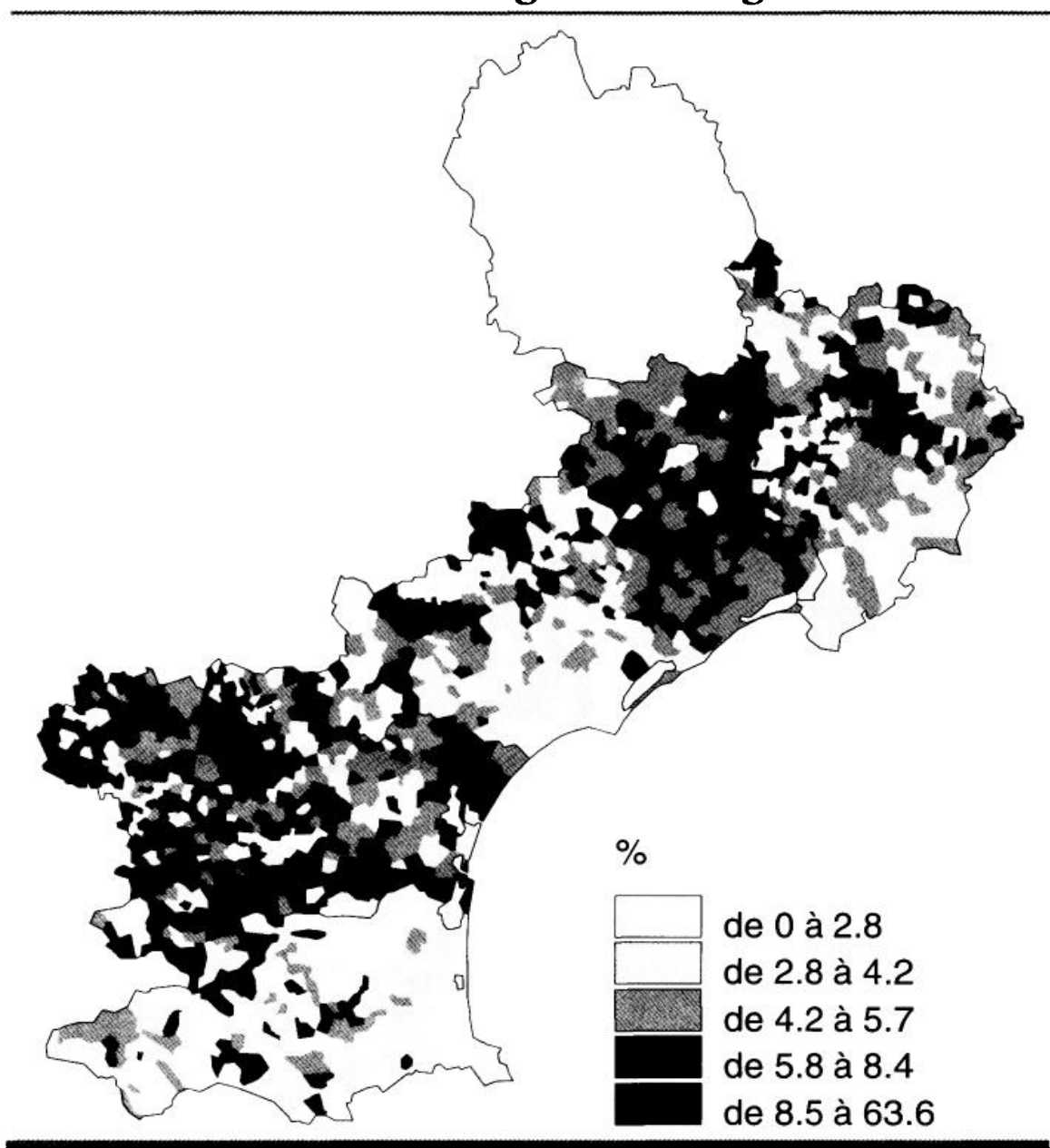

avait fait figure de refuge pour des électeurs de gauche opposés à la liste Allègre, perçue comme un "parachutage" parisien. Cet électorat semble aujourd'hui s'être très largement reporté sur la liste Frêche dans la moitié ouest du département. En revanche dans les espaces de conflit entre ce dernier et Gérard Saumade, Président "divers gauche" du Conseil Général (conflit qui les opposa sur la $4^{c}$ circonscription), et plus largement sur un vaste Montpelliérais, du littoral aux causses, tout porte à croire que l'expression écologiste a fonctionné comme refuge d'une opposition de gauche au maire de Montpellier. La zone

Capcir, Cerdagne et Canigou disparaissent dans la tentative d'autonomisation de Maryse Laperge, qui se solde par un échec retentissant, puisqu'elle perd 12000 des 20000 voix rassemblées en 1992 dans les PyrénéesOrientales. Dans l'Aude et le Gard, les résultats sont plus conformes aux distributions anciennes, celle de 1992 mais également celles de 1988 et 1981 . Le Minervois, Cabardès et surtout le sud des Corbières ont successivement apporté de bons scores à $\mathrm{B}$. Lalonde et A. Waechter. Dans l'Hérault, la situation est rendue complexe par plusieurs effets croisés de listes. En 1992 la liste Génération Écologie, emmenée par Y. Pietrasanta, notable précédemment Radical, d'implantation des écologistes, qui regroupe le nord Montpelliérais, la vallée du Vidourle et les collines sous-cévenoles, devenue traditionnelle, s'en trouve renforcée au sud. La relative concordance entre la géographie de l'influence des écologistes et celle des votes chasseurs pourrait laisser entrevoir de nombreux conflits locaux en termes de contrôle et d'utilisation des espaces naturels ou de loisirs. Les débats sont d'ores et déjà assez vifs à propos des relations entre néo-ruraux, portant un regard "protecteur" sur ces espaces, et couches sociales le contrôlant historiquement et souvent portées à un renforcement identitaire localiste. Le fait que les deux électorats soient fortement volatiles et souvent 


\section{Les élections régionales du 15 mars 1998 en L. $-R$.}

pris dans les incertitudes du "marais" sociopolitique pourrait laisser présager quelques réorganisations surprenantes du paysage politique rural.

\section{Le résistible établissement du Front National}

Le Front National vient de bénéficier en Languedoc-Roussillon d'une légitimation politique assez paradoxale. Consacré comme l'une des composantes majeures de la nouvelle majorité qui a conduit Jacques Blanc à la tête de l'exécutif régional, il marque par ces élections certains signes de repli. Encore faut-il apprécier celui-ci prudemment. Le score global du FN dans la région n'avait cessé d'augmenter depuis les élections européennes de 1994, en atteignant $19 \%$ lors des législatives de 1997. Avec 17,4 \% en 1998 , il retrouve exactement le niveau qui était le sien lors des élections régionales de 1992.

Cependant, la similarité des scores à l'échelle régionale cache d'importants mouvements dans leurs structures départementale et locale (cartes 7 et 8 en annexe).

Le scrutin régional est plutôt défavorable au FN, notamment par rapport aux élections présidentielles et législatives (cf. tableau 1). Bien qu'installé depuis quatorze ans dans l'échiquier politique régional au-dessus de la barre des $10 \%$, il continue d'éprouver certaines difficultés à l'égard d'élections aux enjeux localisés, pour lesquels il ne dispose pas de leadership territorial clair. On en voit la confirmation dans l'exception pyrénéenne de 1992, où le succès du Front National est largement imputable à la personnalité et à l'enracinement local de Pierre Sergent (Arpaillange, Cheylan 1995). Cette même tendance se vérifie, comme on le verra, à l'observation des scores obtenus dans les élections cantonales, souvent encore largement en deçà des niveaux obtenus par cette formation dans les scrutins d'envergure nationale.

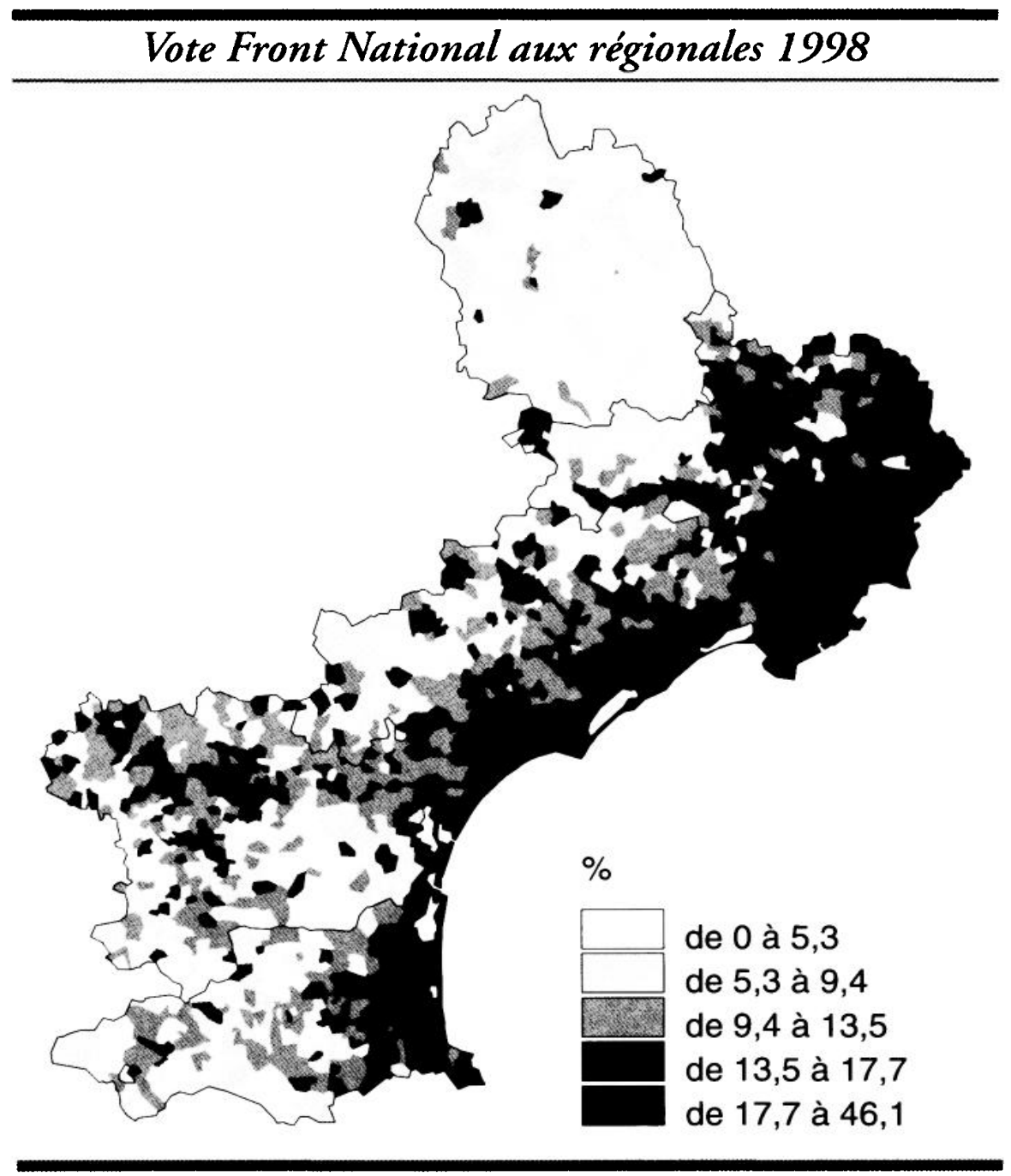




\section{Pôle Sud $N^{\circ} 8$}

\begin{tabular}{|c|c|c|c|c|c|}
\hline & AUDE & GARD & HÉRAULT & LOZĖRE & P-O \\
\hline Européennes 1984 & 8,5 & 12,7 & 15,3 & 6,7 & 15,9 \\
\hline Régionales 1986 & 6,1 & 14,8 & 14,1 & 5,1 & 17,8 \\
\hline Présidentielles 1988 & 13,7 & 20,6 & 19,9 & 11,6 & 20,5 \\
\hline Européennes 1989 & 10,0 & 14,8 & 15,9 & 8,0 & 18,7 \\
\hline Régionales 1992 & 11,6 & 18,2 & 18,3 & 5,9 & 22,6 \\
\hline Législatives 1993 & 11,8 & 17,8 & 14,9 & 5,6 & 18,3 \\
\hline Européennes 1994 & 8,8 & 13,8 & 13,3 & 6,2 & 14,8 \\
\hline Présidentielles 1995 & 13,6 & 20,3 & 19 & 9,6 & 19,5 \\
\hline Législatives 1997 & 14,4 & 21,3 & 19,3 & 8,3 & 21,2 \\
\hline Régionales 1998 & 13,2 & 21,9 & 17,3 & 5,7 & 17,9 \\
\hline
\end{tabular}

2. Évolution 1992-1998 et 1997-1998 (législatives) des scores en voix du F. N. par département (nombre de voix)

\begin{tabular}{lrrrr}
\hline DÉPARTEMENT & 1992 & 1998 & 1997 & 1998 \\
& & & & \\
AUDE & 17450 & +1336 & 22435 & -3619 \\
GARD & 49327 & +4416 & 58170 & -4427 \\
HÉRAULT & 64247 & -3071 & 73350 & -12174 \\
LOZERE & 2542 & -184 & 3321 & -963 \\
PYRÉNÉES-OR. & 36752 & -8333 & 39235 & -10816 \\
& & & & \\
\hline
\end{tabular}

L'Aude manifeste également, comme tous les départements, de tels effets localisés. Dans cette terre emblématique du Midi Rouge et du renouvellement marqué du personnel politique de gauche, le Front National ne cesse pourtant d'accroître son audience. Même si elle reste comparativement plus faible que la moyenne régionale et nationale, elle augmente régulièrement. C'est le département où l'effet "dent de scie" entre scrutins a la plus faible amplitude.

L'Hérault se situe exactement dans la moyenne régionale, deux points au-dessus de la moyenne nationale. Le FN, qui y dispose de certains leaders implantés (Jean-Louis Pelletier à Lunel, Jean-Claude Martinez à Sète), perd $1 \%$ des suffrages, et plus

Derrière ce score régional en demi-teinte, d'importantes disparités sont à relever. Elles sont d'abord le fait de variations départementales assez intéressantes. Comme on le voit, tant en pourcentage des suffrages exprimés qu'en nombre de voix obtenues, l'évolution est en effet contradictoire.

La Lozère est une piètre terre d'élection pour un parti qui ne parvient pas à écorner le leadership de la droite républicaine. Les seules communes $(6$, dont une répond au nom emblématique de "Cultures" !) où le Front National parvient à dépasser en Lozère sa moyenne régionale témoignent plus d'effets locaux (de personnalités figurant sur la liste par exemple) que d'une réelle dynamique politique. de 3000 voix, par rapport à 1992 . C'est sans doute ce dernier, conseiller municipal à Montpellier, qui a été le plus loin, au cours de la campagne, dans la préfiguration d'un accord politique avec Jacques Blanc dans une perspective de conquête future à Sète, avec l'appui en retour de ce dernier. Cette stratégie, vécue comme personnelle au sein du Front National, a pu jeter le trouble au sein même du FN. Toujours est-il qu'elle n'a pas trouvé un large soutien dans les urnes. L'effet de la personnalisation de l'enjeu autour de G. Frêche et de J. Blanc y est sans doute pour quelque chose, de même que le fait qu'une partie des votes FN ait pu cette fois, pour une part, se reporter sur les listes Chasseurs. 


\section{Les élections régionales du 15 mars 1998 en L.-R.}

Les Pyrénées-Orientales témoignent d'un repli encore plus important. Avec un recul de $4,7 \%$ et plus de 8000 électeurs en moins, le FN ne retrouve pas avec A. Jamet, parachuté tête de liste pour l'occasion, les hauts scores dont il était devenu coutumier en terre catalane. Outre qu'il n'a pas su retrouver d'équivalent à la notabilité acquise par P. Sergent, il paye sans aucun doute le prix des difficultés internes consécutives aux problèmes judiciaires rencontrés par son ancien leader, J.-L. Denoël. Plus ici qu'ailleurs encore, le Front National apparaît comme un parti établi, et en cela confronté à des difficultés territoriales "classiques" d'appareil et d'élus.

Le Gard constitue l'autre facette de cet établissement, avec une progression impressionnante vis-à-vis des élections régionales antérieures : $+3,7 \%$ et un gain de près de 4500 voix, assorti pourtant du plus fort taux d'abstention en Languedoc-Roussillon. Outre que ce département représente dans la région son terrain le plus ancien d'implantation (avec Saint-Gilles par exemple), il est aussi celui où s'est construit, succédant à celui de L. De Saint-Affrique, la position dominante de $S$. Martinez. Le succès du chef de file gardois est aussi celui d'une stratégie mégretiste. Fort de son score gardois, il s'est imposé comme le véritable patron du FN à la région.

Les élections régionales de 1992 témoignaient de l'enracinement du FN, et suggéraient la réduction possible des marges de progression du vote frontiste. Cette hypothèse est loin de se vérifier partout. Si l'Hérault et, à un titre différent, les Pyrénées-Orientales semblent la traduction de cet effet de seuil, le Gard et l'Aude démontrent le contraire. Surtout, cette thèse ne résiste pas à deux examens : le rapport entre le vote $\mathrm{FN}$ et le taux d'abstention et, surtout, l'analyse localisée des plus hauts scores enregistrés.

Le recoupement entre les deux cartes (taux d'abstention et vote FN) fait apparaître une correspondance assez remarquable. Si l'on excepte les communes qui bénéficient, par des effets localisés, de forts taux FN (notamment en milieu rural), il s'agit d'un vote qui se situe presque toujours dans les zones de fort abstentionnisme. On peut l'expliquer de deux manières. La première tient au fait que le vote $\mathrm{FN}$ est assez largement un vote urbain et périurbain, celui qui précisément se désintéresse le plus de la chose régionale. La seconde est sans doute la conjugaison de deux phénomènes traduisant la désorientation de l'électorat de droite : le déplacement, sur ces zones, d'une partie de ses électeurs vers un vote extrême, et la défection pure et simple d'une autre partie. Ceci implique que le repli (relatif) du FN par rapport aux élections de 1997 soit moins dû à un sursaut républicain qu'au fait que son électorat potentiel demeure moins facile à mobiliser sur des enjeux régionaux. La réserve de voix frontistes demeure donc élevée. Elle l'est en particulier dans le Gard et dans les P.-O., où une évolution "à la varoise", où le FN s'imposerait comme parti dominant à droite, n'est plus à exclure.

Le FN était enraciné. Il fait désormais partie de l'établissement politique régional. Son implantation est caractérisée par trois phénomènes majeurs. 


\section{Pôle Sud $N^{\circ} 8$}

C'est un vote littoral. Il s'agit là sans doute de sa dimension essentielle, déjà présente lors des élections de 1992, mais qui s'amplifie considérablement. Toutes les communes du littoral, sauf une (Collioure, où persiste une identité politique marquée à gauche, populaire, et peu sensible au développement résidentiel de masse) dépassent leur moyenne départementale. Une grande partie dépasse la moyenne régionale. Ce phénomène est dû pour une partie à l'urbanisation de la côte languedocienne, et à son peuplement consécutif par des catégories de population âgées, sensibles à la thématique sécuritaire, et dont les réseaux sociaux locaux sont peu denses. C'est la facette bourgeoise du vote frontiste, qui trouve sa parfaite correspondance dans la progression du FN dans les communes périurbaines autour de Béziers, Montpellier, Nîmes et Perpignan, toutes typiques de l'urbanisation en lotissements issus de la politique d'accession massive à la propriété individuelle. Il y a d'ailleurs une concomitance entre l'extension des politiques de lotissement et la progression du vote $F N$, visible notamment dans l'axe Saint-Mathieu de Tréviers/Quissac.

C'est également un vote urbain. Les hypothèses qui tendaient à la ruralisation du Front National sont assez largement démenties par un fait majeur : sauf exceptions de peu de portée, c'est dans et autour des villes que le FN s'établit avec le plus de force. C'est vrai des grandes villes, mais aussi des villes moyennes comme Bagnolssur-Cèze, Aigues-Mortes, La GrandCombe ou Pont-Saint-Esprit dans le Gard, Agde, Sète, Lunel et Lodève dans l'Hérault, Limoux ou Castelnaudary dans l'Aude.
C'est moins vrai dans les P.O. et en Lozère. La ruralisation particulière que pouvait représenter une assez forte progression du vote $\mathrm{FN}$ dans les anciennes communes "pinardières" (définies par les zones d'arrachage subventionné massif de vignes) n'est pas valable partout : on la retrouve dans les scores situés autour de Béziers (Maraussan, Puissalicon, Espondeilhan et la zone située entre Béziers et Saint-Chinian, non-compris). Mais il s'agit sans doute plus d'une progression en étoile d'un comportement périurbain du Biterrois qu'une conversion claire de l'ancienne société viticole, aux thèses frontistes. Il faut cependant noter que, dans cette même zone, le vote communiste, qui représentait une dimension considérable du comportement de cette société, accentue encore son déclin.

C'est un vote de rejet de certaines des évolutions économiques et sociales globales. L'assimilation du vote $\mathrm{FN}$ à un vote protestataire, au sens où celui-ci ne ferait qu'exprimer un ras-le-bol général sans consistance politique, ne résiste pas au constat de la stabilisation de son électorat, et au fait que celui-ci s'exprime désormais en parfaite connaissance de cause. De plus, l'offre alternative de vote protestataire, de défoulement, existait largement (à travers les listes de Chasseurs, de défenseurs du Roussillon, des contribuables ou de l'"apolitisme" en politique). Mais ceci n'est pas contradictoire avec le fait que l'on trouve des scores FN très élevés dans les communes marquées par des symptômes aigus de crise : la carte du Gard recoupe fortement celle de la reconversion industrielle, de même que celle de l'Hérault. Le couloir pyrénéen vers l'Espagne, sinistré à cause de 


\section{Les élections régionales du 15 mars 1998 en L.-R.}

la suppression des frontières et de l'économie qu'elles généraient, a la même coloration. Lodève, Lunel, Limoux, et Frontignan ont des caractéristiques similaires. Cependant, le Front National éprouve parfois des difficultés à capter cet électorat. C'est le cas d'Alès, où le FN ne parvient ni à atteindre la moyenne gardoise, ni celle de la région. Ici, le vote communiste, qui demeure élevé, constitue encore la voie majeure, enracinée et légitime de contestation à l'égard des effets de la désindustrialisation. Dans le cas du Vigan, c'est le vote socialiste qui se maintient à un niveau élevé, avec un vote $\mathrm{FN}$ inférieur à $10 \%$.

Enfin, cet établissement du Front National reste freiné par la question, cruciale au sein du parti, de la légitimation locale. On notera que les candidats FN sont, notamment aux élections cantonales, souvent peu ou pas connus, que la structuration municipale, en dehors des scrutins, est souvent inexistante. C'est sans doute ce qui explique la difficulté pour le $\mathrm{FN}$, dans bien des endroits, à conduire son électorat à la logique des reports de voix (ladite "discipline nationale" de J.-M. Le Pen). Dans une grande majorité des cas, les scores obtenus aux élections régionales par les listes FN sont très largement supérieurs à ceux, obtenus le même jour, par le candidat local du FN dans les cantons en renouvellement. Les exceptions consacrent toujours la présence d'un leader local connu, disposant souvent d'un autre mandat (conseiller municipal ou régional par exemple) : Roudil à Alès, Janin à Sommières, Lépine, Santoyo, S. Martinez à Nîmes, J.-C. Martinez à Sète, J.-L. Pelletier à Lunel... On notera toutefois que la stratégie d'asso- ciation à la majorité conduite à l'échelle régionale par J. Blanc n'a pas entraîné, pour ceux de ces leaders, les reports de voix suffisants pour une élection de conseiller général qui reste la plus difficile pour le FN. Les scores de ses candidats ne progressent pas significativement entre les deux tours en cas de maintien, même seuls face à la gauche. La résistance à l'établissement du FN trouve sans doute ici sa plus claire illustration.

Les élections régionales de 1998 n'ont pas provoqué le bouleversement, parfois attendu, des équilibres entre forces politiques. Au-delà des mutations, souvent importantes, qui affectent telle ou telle partie de la région ou de ses courants politiques, la stabilisation du rapport de force entre droite et gauche est l'un des enseignements de ce scrutin. C'est notamment le résultat logique (en contraste très net avec les législatives de 1997) d'un mode de scrutin proportionnel. Mais ce fait est d'autant plus remarquable que, pour la première fois, ces élections se déroulaient dans un contexte plutôt favorable à la gauche. En 1986 , la nationalisation du vote (associé aux législatives le 16 mars 1986) laissait supposer un basculement à droite. En 1992, la délégitimation du gouvernement socialiste faisait de ces élections un premier tour de chauffe de l'alternance qui suivrait un an plus tard. Force est de constater que la dimension nationale des comportements électoraux n'a pas, en LanguedocRoussillon, inversé une tendance à l'équilibre des rapports de force. Toutefois, il 


\section{Pôle Sud $N^{\circ} 8$}

faut tout de suite corriger ce constat général par trois remarques.

La progression de l'abstention d'un scrutin à l'autre, même si son taux reste cette fois inférieur à la moyenne nationale, suggère que, faute d'être tirée vers le haut par un contexte national spécifique, l'élection des Conseils Régionaux demeure intermédiaire et mobilise peu.

Ensuite, cette stabilité politique relative va de pair avec un taux de renouvellement très important du personnel politique régional. C'était déjà le cas en 1992, où $63 \%$ des élus régionaux étaient nouveaux (Darviche, Genieys, Joana 1995). En 1998, c'est à nouveau et exactement ce même chiffre. Cette discontinuité du personnel politique, qui laisse peu de place à l'établissement (en dehors sans doute du Président et de quelques personnalités) d'une véritable carrière politique régionale, rend également problématique l'idée qu'une classe politique régionale serait en cours d'émergence. Au-delà de certains traits sociologiques en termes d'âge, de filière militante, et de catégorie socioprofessionnelle, rien ne vient définir les conditions minimales (institutionnalisation, autonomisation, continuité des carrières) pour parler d'une véritable classe politique originale. Cela est d'autant plus vrai lorsque l'on compare la situation languedocienne avec sa voisine catalane où, par contre, on retrouve de tels critères : un taux de renouvellement bien moindre et régulier, un ancrage singulier sur le territoire, une différenciation institutionnelle plus nette sont les marques de cette classe politique (Genieys 1998). Or de telles différences entre régions appelées depuis plus de dix ans à coopérer et à affron- ter des enjeux similaires, au moins formellement, sont loin d'être négligeables. On peut notamment expliquer par cette différence de continuité des carrières et du personnel politiques les variations importantes qui existent de part et d'autres des Pyrénées quant à l'existence et à la cohérence de l'espace public régional. C'est sans doute aussi pour cette raison que, même s'il est frappant de constater des similitudes entre les deux personnalisations du leadership catalan (PujolMaragall) et languedocien (Frêche-Blanc), les enjeux politiques régionaux demeurent très étrangers l'un à l'autre.

Enfin, le relatif affaiblissement de la droite "notabiliaire" laisse le champ libre à un établissement, qui n'a sans doute pas encore atteint son plein rendement, du Front National. Pour être confronté, dans les élections d'envergure locale, à un problème d'enracinement supérieur à la droite classique, le FN n'en est pas moins en train de capitaliser les effets de certaines formes de notabilisation. C'est particulièrement clair dans le Gard. La stratégie mégretiste ne s'est pas traduite pleinement, notamment par la reproduction de pratiques de parachutage d'élus dans des circonscriptions toujours différentes. Là où elle prend de la consistance (la Petite Camargue, le Gard en général), les scores aux élections locales progressent nettement. On peut se demander comment la droite classique, dont les leaders montrent localement d'inquiétants signes de faiblesse, répondrait à une systématisation de l'implantation locale (hors élections par exemple) des représentants du FN. À l'appui de cette stratégie, la légitimation que procure au FN son intégration par Jacques Blanc dans 


\section{Les élections régionales du 15 mars 1998 en L. $-R$.}

la majorité présidentielle régionale pourrait se traduire, pour la droite, par des effets contradictoires.

Dans une conjoncture électorale somme toute relativement stable, deux questions se trouvent paradoxalement posées aux grands blocs Gauche-Droite :

- passée d'une fragmentation départementale historique à une synergie d'action régionale, la gauche saura-t-elle conserver cette ligne d'action après son échec aux régionales et son repli sur les Conseils Généraux?
- désormais coupée des appareils nationaux, la droite locale devra trouver sa voie face à la stratégie de la "main tendue" des mégretistes, qui, en l'absence de contre-poids sérieux de l'UDF et du RPR, peuvent faire du Languedoc-Roussillon un terrain d'expérimentation de "l'alliance nationale" plus discret et courtois que celui ouvert chaotiquement en Provence-Alpes-Côte d'Azur. Alors que la transformation sociologique et économique de cette région se poursuit en accéléré, les formes partisanes pourraient, elles aussi, rentrer durablement en mutation.

\section{Notes}

1. "L'état du Languedoc-Roussillon ", Revue de l'Economie Méridionale, n¹37, 1987. " Midi Rouge? Midi bouge ", Amiras, n¹7, octobre 1987. "Languedoc-Roussillon 1975-1995: la mutation sociale et structurelle ", Le Magazine économique et financier du Languedoc-Roussillon, ${ }^{\circ} 20$, décembre 1997.

2. Midi Libre, le 30/07/1997.

3. Midi Libre, le 05/09/1997, p. 3.

4. Midi Libre, le 05/09/1997, p. 3.

5. Sur l'opposition structurelle entre le maire de Montpellier et le Président du Conseil Général de l'Hérault au sein du Parti Socialiste, voir Arpaillange, 1994.

6. La Gazette de Montpellier, $n^{\circ}$ 518, mars 1998, p. 14.

7. La Gazette de Montpellier, $\mathrm{n}^{\circ} 518$, mars 1998, p. 15.

8. Ce qui représente un gain de $2,75 \%$. Outre la progression liée aux flux démographiques positifs de la région, cette augmentation est également liée à l'inscription automatique des jeunes électeurs âgés de plus de 18 ans, automatisation en vigueur depuis fin 1997.

9. Telle qu'elle a été proposée par le Président socialiste du Conseil Général du Gard à ses trois nouveaux collègues, pour " isoler " la région et assurer une coordination propre des politiques départementales à l'échelon régional, notamment par le boycott du Contrat de Plan État-Région. 


\section{Pôle Sud $N^{\circ} 8$}

\section{Références}

Amiras, "Midi rouge?... Midi bouge. Les élections de mars 1986", Repères Occitans, numéro spécial, 1986.

Alliès, P., "Un midi en voie de banalisation politique?", Pôle Sud, N², 1995.

Arpaillange, C., Une économie si politique. Développement économique et conflictualité politique dans l'Hérault, Mem. DEA Science Politique, I.E.P. Bordeaux, 1994.

Birnbaum, P., La France imaginé, Paris, Fayard, 1998.

Bon, F.; Cheylan, J.-P., La France qui vote, Paris, Hachette (Pluriel), 1988.

Arpaillange, C., Cheylan, J.-P., "Les élections de mars 1992 en Languedoc-Roussillon : entre nationalisation des votes et restructuration du système notabiliaire", Pôle Sud, $\mathrm{N}^{\circ} 2,1995$.

Darviche, S., Genieys, W., Joana, J., "Sociologie des élus régionaux en Languedoc et en Pays de Loire", Pôle Sud, N², 1995 ..

Genieys, W., "Élites intermédiaires et frontières institutionnelles : une comparaison Catalogne et LanguedocRoussillon", Revue Européenne des Migrations Internationales, 13 (3), 1997.

Parodi, J.-L., "La double consultation de mars 1992. À la recherche d'un modèle", in Habert, P., Perrineau, P., Ysmal, C., Le vote éclaté. Les élections régionales et cantonales des 22 et 29 mars 1992, Paris, Presses de la FNSP, 1992.

Ritaine, E., Changement social et pratiques développementalistes en Languedoc, Étude pour le Ministère de la Recherche et de la Technologie, CERVL-IEP Bordeaux, 1991.

Ritaine, E., "La modernité localisée? Leçons italiennes sur le développement local", Revue Française de Science Politique, 39 (2), 1989. 


\section{Annexes \\ Pourquoi des cartes d'évolution des votes et des cartes de résidus de régression*?}

Lorsque l'on examine les répartitions géographiques de plusieurs résultats successifs d'une même expression politique, de fortes ressemblances apparaissent, traduisant les différences régionales d'implantation du courant, relativement permanentes. Cette ressemblance peut être statistiquement mesurée par le coefficient de corrélation. Il permet, à partir du premier résultat, de calculer des "valeurs attendues" pour le second si le comportement de tous les lieux avait varié de la même façon. Les résultats effectifs au deuxième scrutin s'écartent quelque peu de ces valeurs attendues, et ces écarts constituent, pour chaque unité observée, le "résidu de la corrélation". Ils sont fortement significatifs de différences locales de comportement entre les deux scrutins, mettant en évidence les dynamiques différentielles dans l'espace, qu'elles soient dues à des effets de liste (ici, par exemple, de fortes différences entre les comportements des départements) ou de personnalité dans leur aire d'influence (par exemple, le cas des votes Génération Écologie en 1992, fortement influencés par l'implantation du maire de Mèze, Y. Pietrasanta), ou encore à des différences régionales d'ordre historique ou socioculturel (La Cévenne protestante vote presque toujours plus à gauche que ne le laisserait penser l'évolution d'ensemble). Les cartes de résidus livrent donc au lecteur l'intensité et la répartition géographique de ces différences.

\footnotetext{
* Nous remercions Bruno Granier et le Service informatique du MIdi Libre pour leur précieuse collaboration en matière de recueil des données électorales.
} 


\section{Pôle Sud $N^{\circ} 8$}

1. Élections régionales de 1992 et de 1998 Taux d'évolution des votes de droite

2. Résidus de régression régionale 98 /régionale 92 Évolution des votes de droite

3. Elections régionales de 1992 et de 1998 Taux d'évolution des votes de gauche

4. Résidus de régression régionale 98 /régionale 92 Évolution des votes de gauche

5. Élections régionales de 1992 et de 1998 Taux d'évolution des votes Chasse

6. Élections régionales de 1992 et de 1998 Taux d'évolution des votes Verts

7. Élections régionales de 1992 et de 1998 Taux d'évolution des votes du Front National

8. Résidus de régression régionale $98 /$ régionale 92 Évolution des votes du Front National 
Les élections régionales du 15 mars 1998 en L.-R.

Élections régionales de 1992 et de 1998

Carte 1 : Taux d'évolution des votes de droite

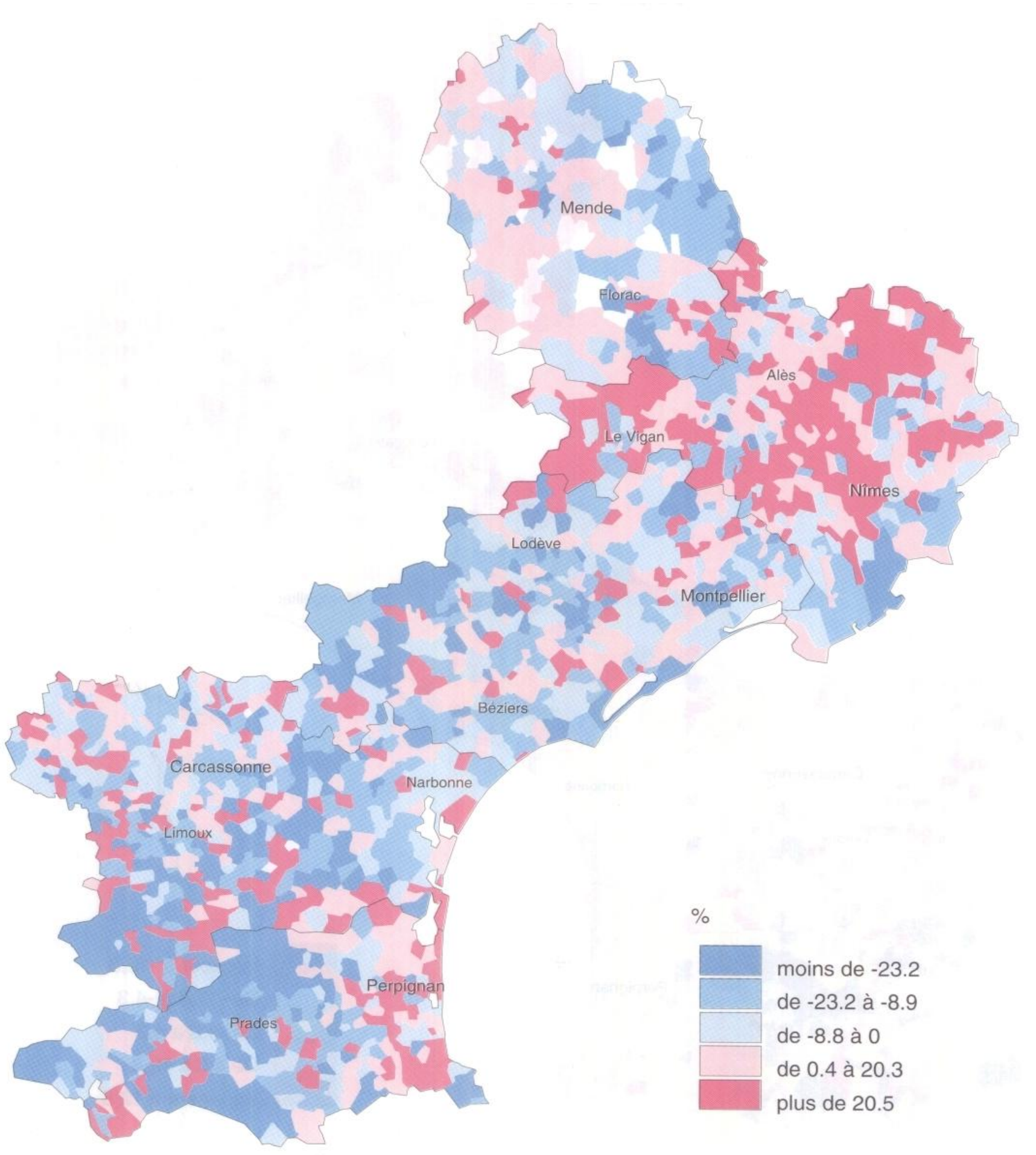




\section{Pôle Sud $N^{\circ} 8$}

Résidus de régression régionale 98/régionale 92

Carte 2 : Évolution des votes de droite

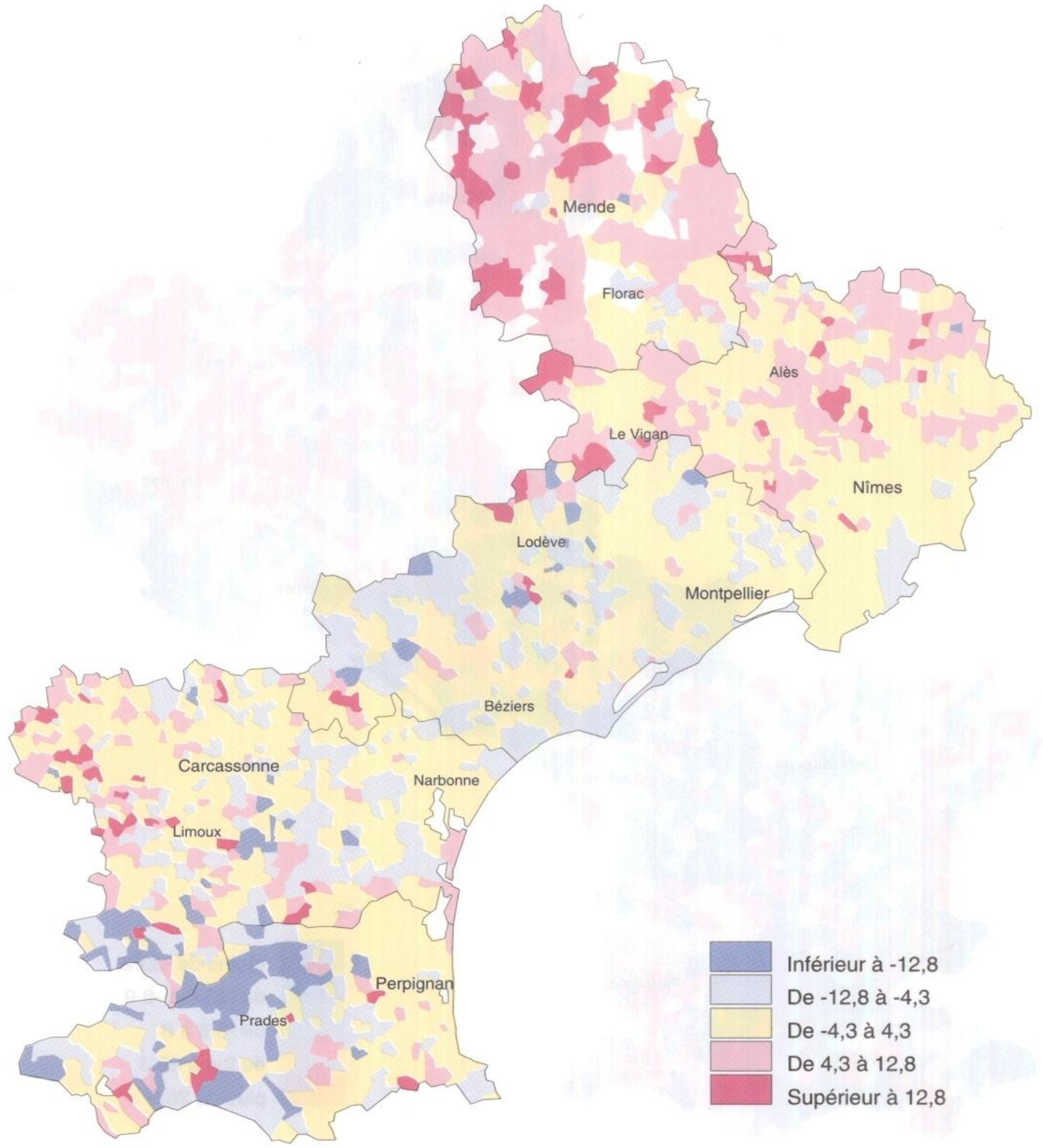




\section{Les élections régionales du 15 mars 1998 en L.-R.}

\section{Élections régionales de 1992 et de 1998}

Carte 3 : Taux d'évolution des votes de gauche
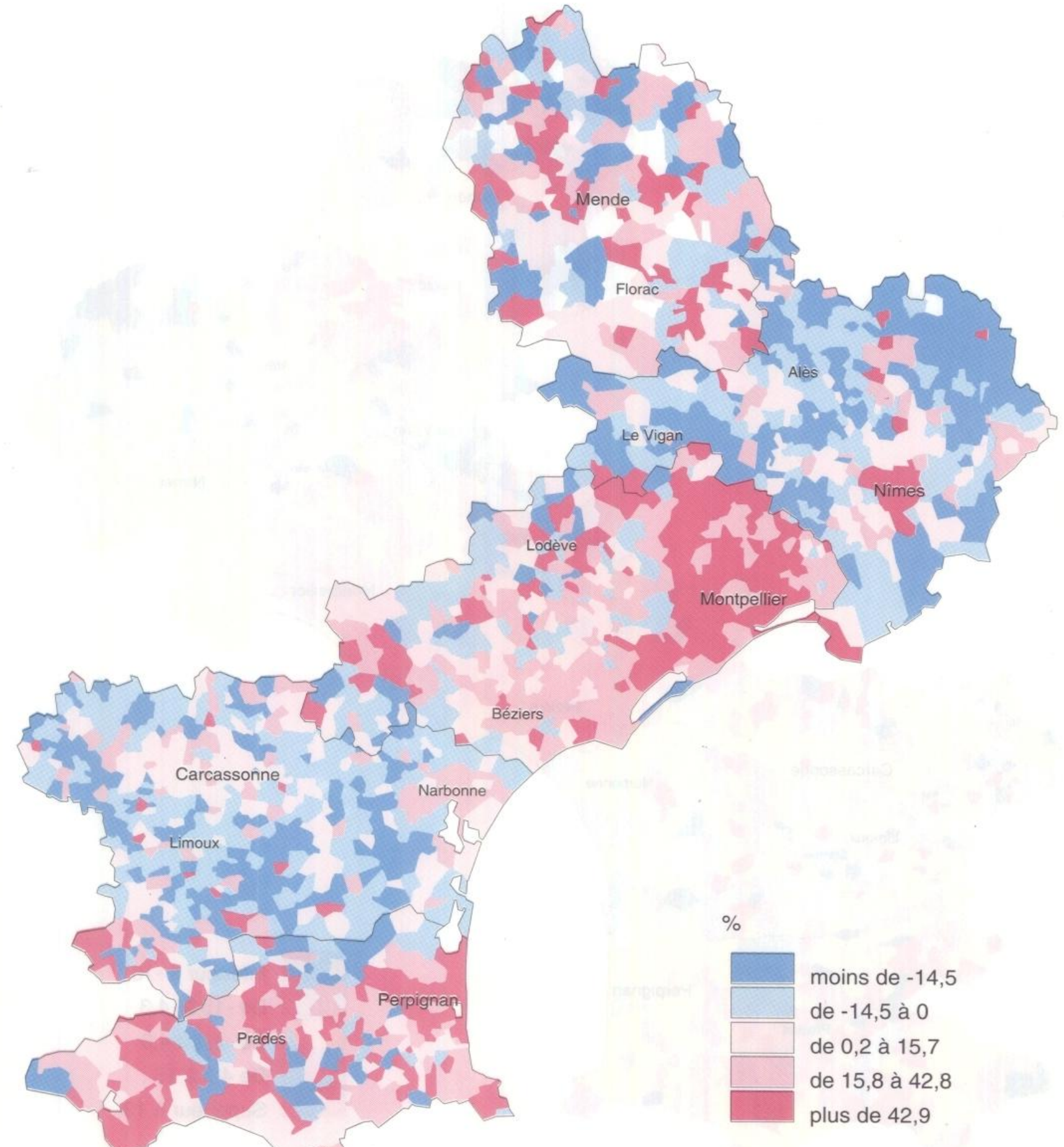


\section{Pôle Sud $N^{\circ} 8$}

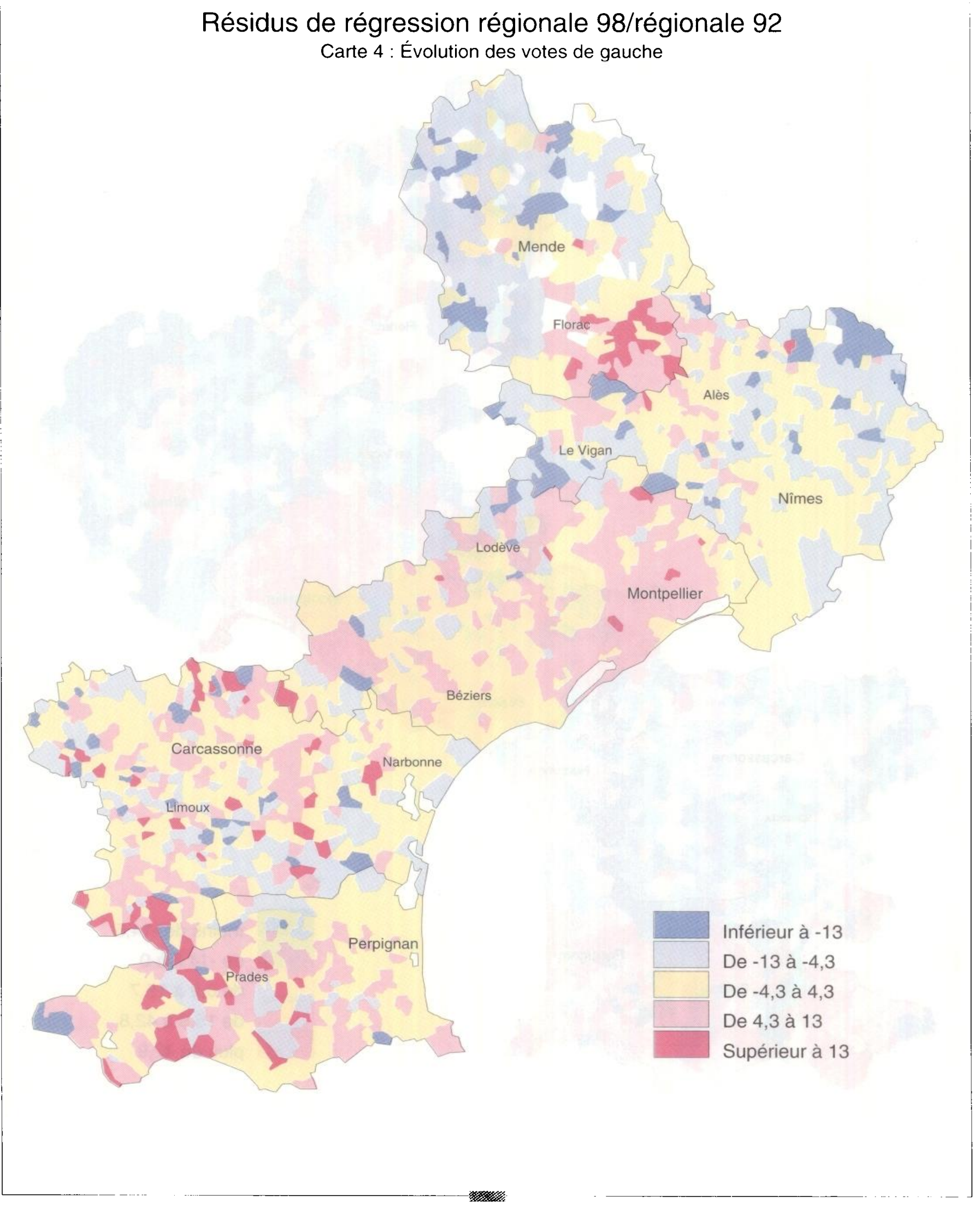


Les élections régionales du 15 mars 1998 en L. $-R$.

Élections régionales de 1992 et de 1998

Carte 5 : Taux d'évolution des votes "chasse"

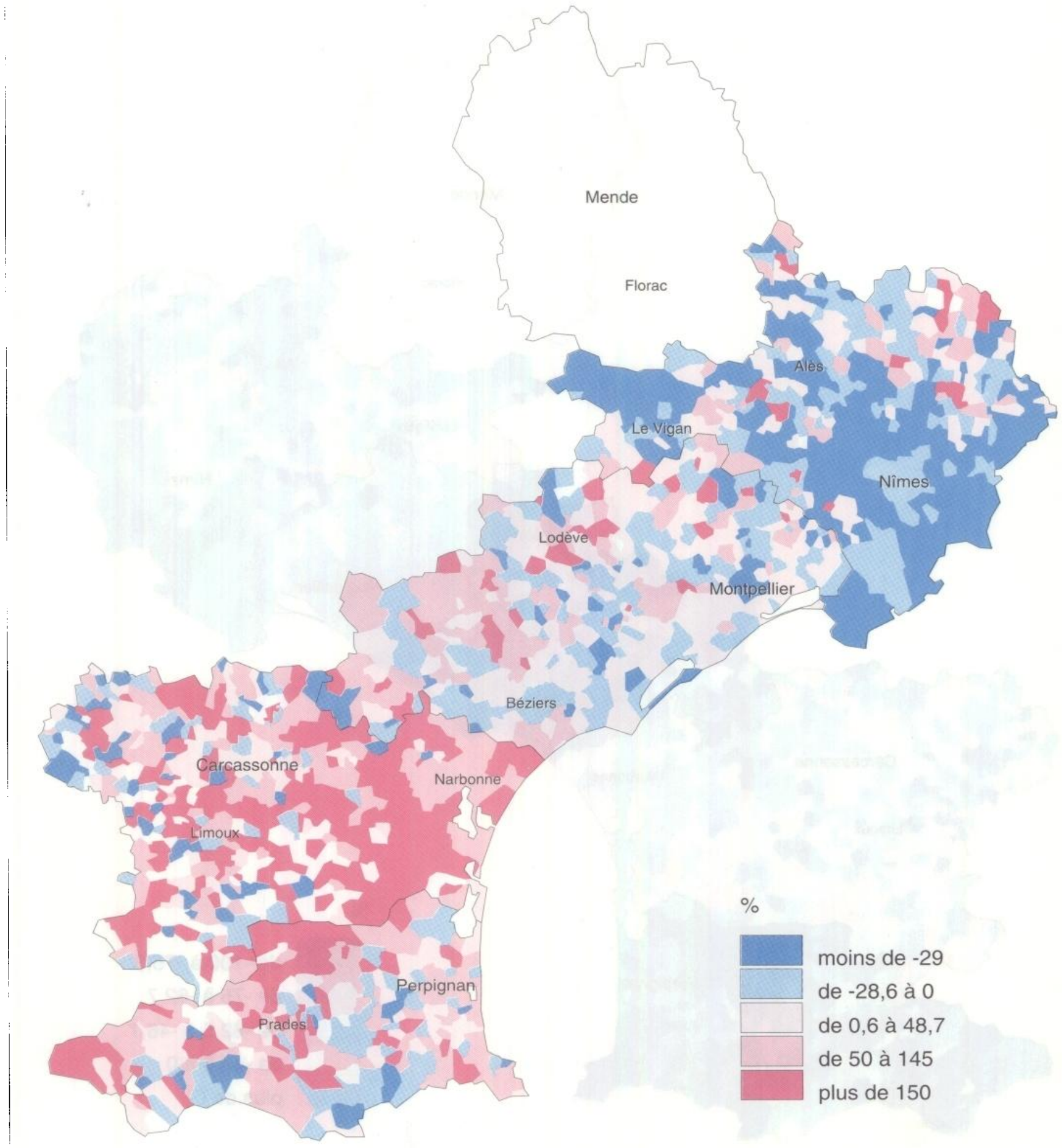




\section{Pôle Sud $N^{\circ} 8$}

Élections régionales de 1992 et de 1998

Carte 6 : Taux d'évolution des votes verts

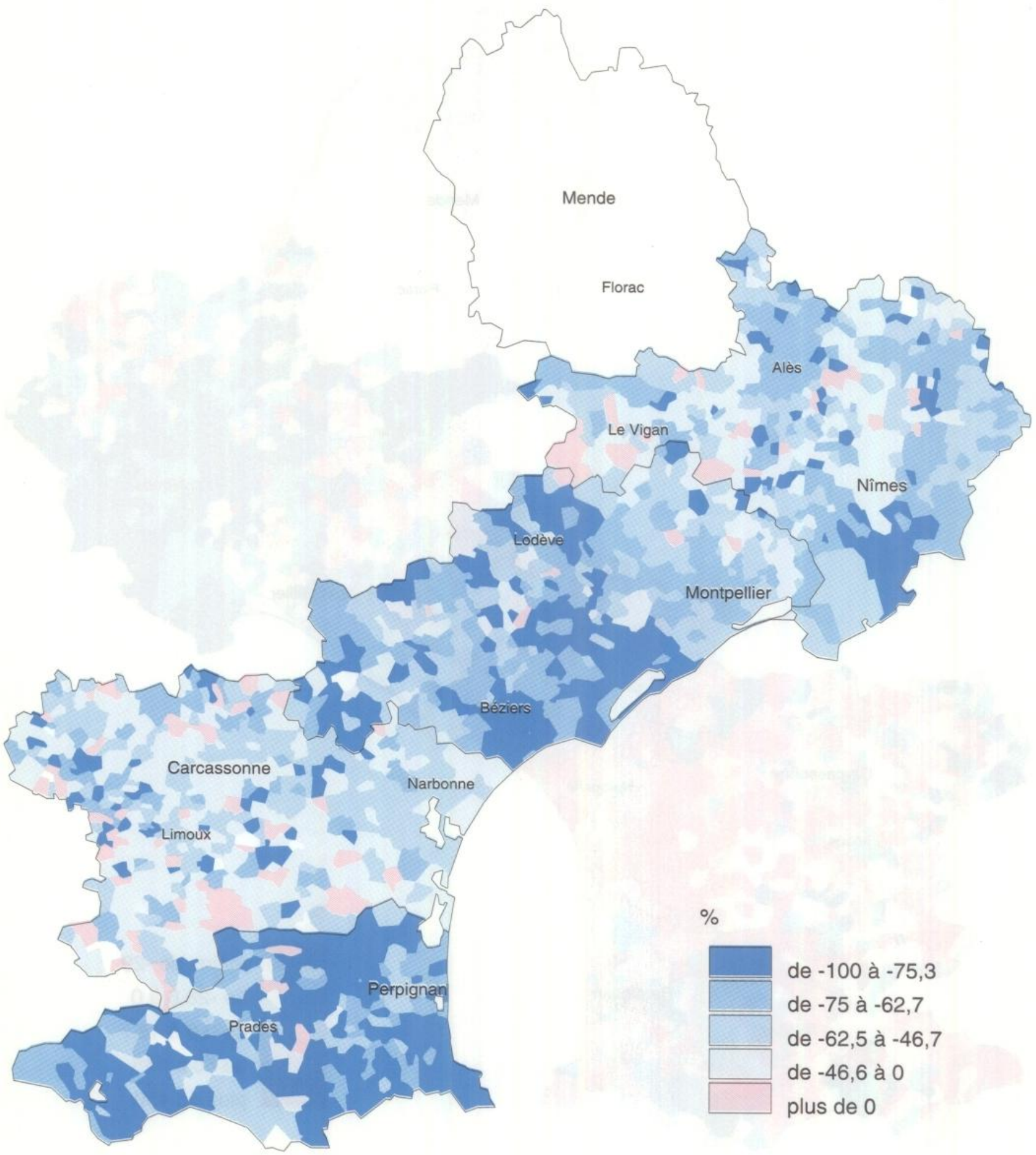


Les élections régionales du 15 mars 1998 en L. $-R$.

Élections régionales de 1992 et de 1998

Carte 7 : Taux d'évolution des votes du front national

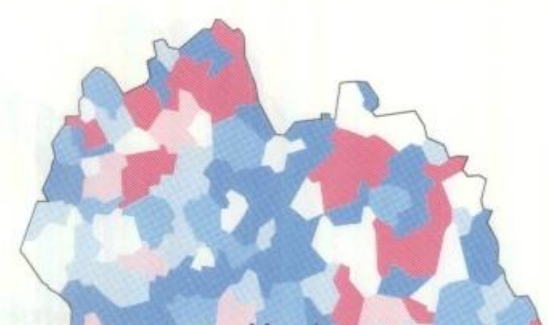

1 Mendery

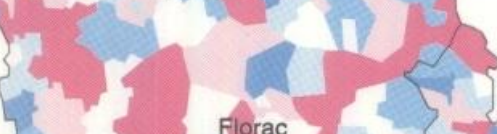

$\int_{\text {Florac }}$ 


$$
\text { Pôle Sud } N^{\circ} 8
$$

\section{Résidus de régression régionale 98/régionale 92}

Carte 8 : Évolution des votes du front national

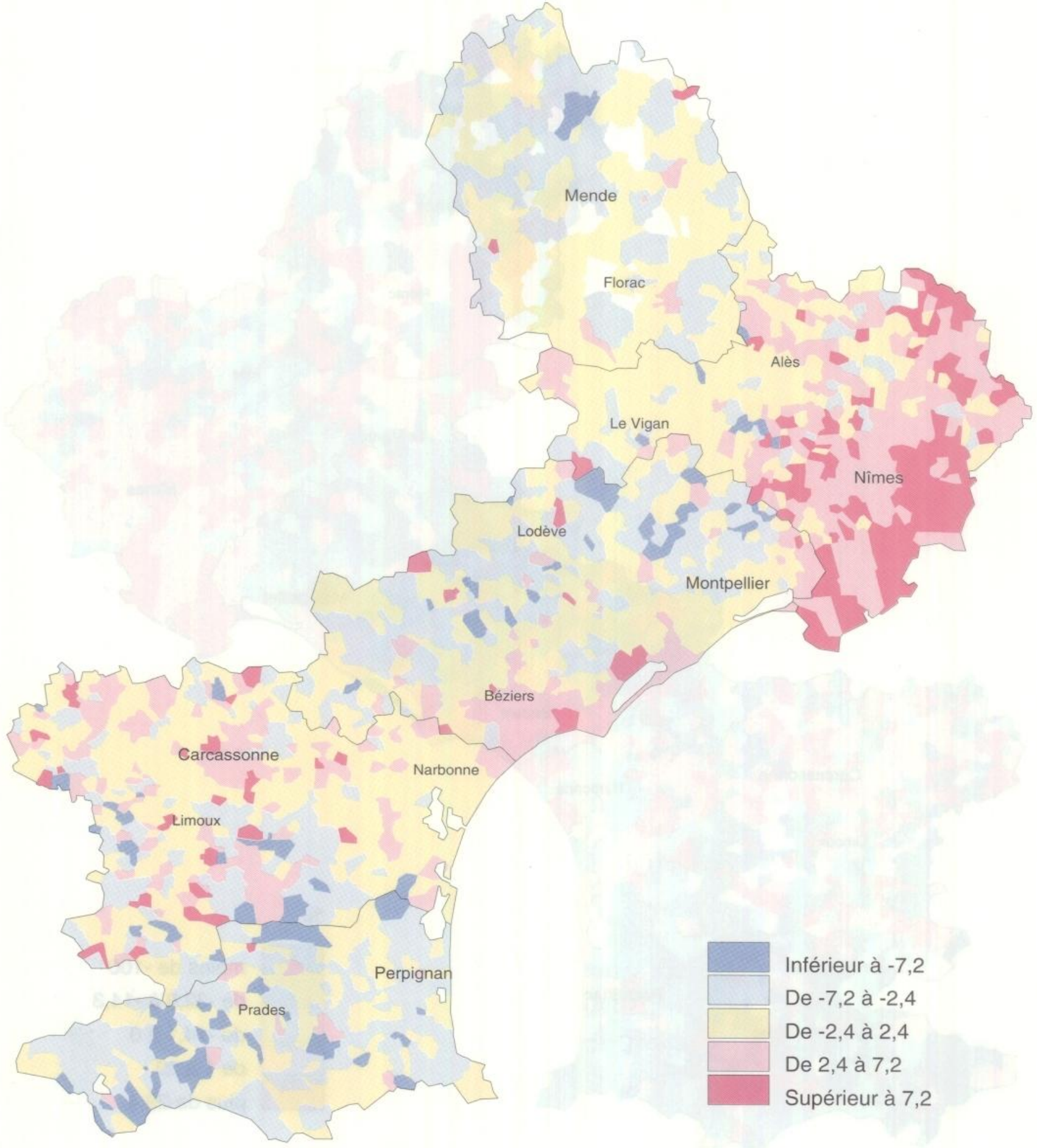

